\title{
Mapping properties of fundamental operators in harmonic analysis related to Bessel operators
}

\author{
by \\ Jorge J. Betancor (La Laguna), Eleonor Harboure (Santa Fe), \\ Adam Nowak (Wrocław) and Beatriz Viviani (Santa Fe)
}

\begin{abstract}
We obtain sharp power-weighted $L^{p}$, weak type and restricted weak type inequalities for the heat and Poisson integral maximal operators, Riesz transform and a Littlewood-Paley type square function, emerging naturally in the harmonic analysis related to Bessel operators.
\end{abstract}

1. Introduction. In his monograph [16] Stein suggested the study of analogues of the fundamental operators in the classical harmonic analysis, such as Riesz transforms, conjugate Poisson integrals, multipliers, fractional integrals, maximal functions, square functions, in a context of discrete or continuous expansions with respect to eigenfunctions of self-adjoint and positive differential operators. During the last years, this program, or some of its aspects, has been successfully developed by many authors in various settings.

The study in the framework of Bessel (and also ultraspherical) operators was initiated even before [16] by the seminal paper [13] of Muckenhoupt and Stein. They introduced the notion of conjugation in the Bessel setting, and their starting point was the formulation of suitable Cauchy-Riemann type equations leading to a definition of conjugate Poisson integrals. Then the Riesz transform, or rather the conjugate function mapping according to the terminology used in [13, emerges as the corresponding boundary value. After [13] the Bessel context was investigated by several authors. In particular, recently Betancor and Stempak [6] and Betancor, Buraczewski, Fariña, Martínez and Torrea [3, 4] obtained some boundedness results for a Riesz transform and $g$-functions in Bessel settings.

The aim of the present paper is to advance the study of $L^{p}$ mapping properties of several basic operators related to the harmonic analysis of Bessel

2010 Mathematics Subject Classification: Primary 42C05; Secondary 42C20.

Key words and phrases: Bessel operator, heat-diffusion integral, Poisson integral, maximal operator, Riesz transform, square function. 
operators. We analyze the behavior of the maximal operators for the heat and Poisson integrals, Riesz transform and a $g$-function (see Section 2 for rigorous definitions of these objects) associated with the Bessel operator appearing in [13,

$$
\Delta_{\lambda}=-\frac{d^{2}}{d x^{2}}-\frac{2 \lambda}{x} \frac{d}{d x}, \quad \lambda>-1 / 2,
$$

which is formally self-adjoint in $L^{2}\left(\mathbb{R}_{+}, d \mu_{\lambda}\right)$ with $\mathbb{R}_{+}=(0, \infty)$ and

$$
d \mu_{\lambda}(x)=x^{2 \lambda} d x, \quad x>0 .
$$

Our main interest is focused on characterizing the power weights $x^{\delta}$ for which the above-mentioned operators are of strong type, weak type or restricted weak type $(p, p)$ with respect to the measure $x^{\delta} d x$. We shall give a complete description of such power weights, and in all cases prove the outcomes to be sharp.

Our results are achieved by the nowadays standard method of splitting the integral kernels into local and global parts, where local is related to a symmetric cone containing the diagonal of $(0, \infty) \times(0, \infty)$. Following Muckenhoupt and Stein [13], we show that in the local region the operators behave like those derived from the usual Laplacian, while in the global region they are essentially controlled by Hardy-type operators. In order to get sharp results for the range of the power weights, it is necessary to obtain a precise knowledge of the behavior of the kernels involved. We use several tools in performing this task, one of them being the local Calderón-Zygmund theory established in [14.

We point out that in the literature there are some recent related results regarding the harmonic analysis derived from the Bessel operator

$$
\widetilde{\Delta}_{\lambda}=-\frac{d^{2}}{d x^{2}}-\frac{\lambda(1-\lambda)}{x^{2}},
$$

which is formally self-adjoint in $L^{2}((0, \infty), d x)$. In particular, in [3, 4, 6] Riesz transforms and $g$-functions were studied in this setting. The results contained in the present paper have counterparts in the framework of $\widetilde{\Delta}_{\lambda}$. Moreover, for proving those twin results there is no need to carry out parallel computations since we may directly take advantage of the estimates and properties already shown in the $\Delta_{\lambda}$ context. Comments sketching how the corresponding results in the $\widetilde{\Delta}_{\lambda}$ setting can be deduced will be given along the paper.

Finally, let us give a short account of the previous results concerning the operators we investigate. For the Poisson and heat-diffusion integrals, the unweighted case, with the restriction $\lambda>0$, was studied in [13] and [7], respectively. A $g$-function based on the Poisson kernel was investigated in [17, where strong type $(p, p)$ for $p>1$, with respect to the measure $\mu_{\lambda}, \lambda>0$, 
was obtained. Considering the Riesz transform, in [1] a characterization of the weights for strong type $(p, p), 1<p<\infty$, and weak type $(1,1)$ was given. The approach to this operator was analogous to that in [13, through conjugate Poisson integrals. Here we adopt the point of view taken in [3, 4], and show that for any $\lambda>-1 / 2$ the Riesz transform is a principal value integral with a kernel that satisfies similar estimates to those in [1]. Although the scope of [1] for the strong and weak type inequalities is more general than ours, we also analyze restricted weak type obtaining new weighted inequalities.

The paper is organized as follows. In Section 2 we introduce the main objects of our study and state the main results, Theorems 2.1 2.5. There we also gather some general facts and lemmas that will be used throughout the paper. The remaining Sections 36 are separately devoted to the heat integral maximal operator, Riesz transform, $g$-function and Poisson integral maximal operator, respectively, and the proofs of the main theorems. In Section 6 we also take into account a square function related to the Poisson integral.

2. Preliminaries and statement of results. Recall that the standard set of eigenfuctions of the Bessel operator $\Delta_{\lambda}$ consists of

$$
\varphi_{z}^{\lambda}(x)=(z x)^{-\lambda+1 / 2} J_{\lambda-1 / 2}(z x), \quad x, z>0,
$$

where $J_{\nu}$ is the Bessel function of the first kind and order $\nu>-1$. Indeed, a straightforward computation (cf. [12, Section 5.2]) shows that, for $\lambda>-1 / 2$,

$$
\Delta_{\lambda} \varphi_{z}^{\lambda}=z^{2} \varphi_{z}^{\lambda}, \quad z>0 .
$$

Thus the heat kernel associated to $\Delta_{\lambda}$ is

$$
W_{t}^{\lambda}(x, y)=\int_{0}^{\infty} e^{-z^{2} t} \varphi_{z}^{\lambda}(x) \varphi_{z}^{\lambda}(y) d \mu_{\lambda}(z), \quad t, x, y>0 .
$$

Computing the last integral (see [18, p. 395]) leads to

$$
W_{t}^{\lambda}(x, y)=\frac{(x y)^{-\lambda+1 / 2}}{2 t} e^{-\left(x^{2}+y^{2}\right) / 4 t} I_{\lambda-1 / 2}\left(\frac{x y}{2 t}\right), \quad t, x, y>0,
$$

with $I_{\nu}$ being the modified Bessel function of the first kind and order $\nu>-1$. Then the heat-diffusion integral of a function $f$ is defined by

$$
W_{t}^{\lambda} f(x)=\int_{0}^{\infty} W_{t}^{\lambda}(x, y) f(y) d \mu_{\lambda}(y), \quad t, x>0 .
$$

Denote by $W_{*}^{\lambda}$ the corresponding maximal operator,

$$
W_{*}^{\lambda} f=\sup _{t>0}\left|W_{t}^{\lambda} f\right| .
$$


Our result concerning $W_{*}^{\lambda}$ is the following (see also Figure 1 below).

Theorem 2.1. Let $\lambda>-1 / 2,1 \leq p<\infty, \delta \in \mathbb{R}$. Then the maximal operator $W_{*}^{\lambda}$, considered on the measure space $\left(\mathbb{R}_{+}, x^{\delta} d x\right)$, has the following mapping properties:

(a) $W_{*}^{\lambda}$ is of strong type $(p, p)$ iff $p>1$ and $-1<\delta<(2 \lambda+1) p-1$;

(b) $W_{*}^{\lambda}$ is of weak type $(p, p)$ iff $-1<\delta<(2 \lambda+1) p-1$ or $\delta=2 \lambda$;

(c) $W_{*}^{\lambda}$ is of restricted weak type $(p, p)$ iff $-1<\delta \leq(2 \lambda+1) p-1$. Moreover, $W_{*}^{\lambda}$ is of strong type $(\infty, \infty)$.

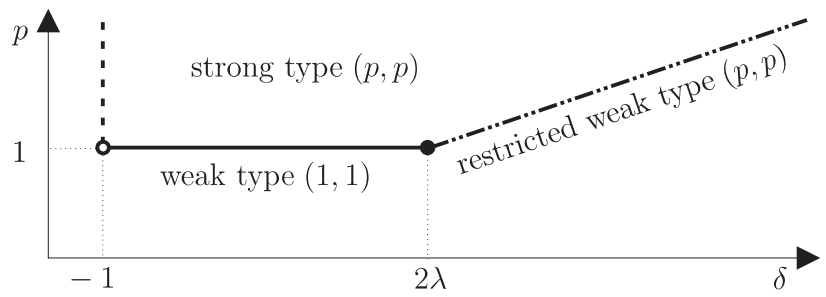

Fig. 1. Mapping properties of $W_{*}^{\lambda}($ fixed $\lambda=1)$

According to 2.1, the Poisson kernel is given by

$$
P_{t}^{\lambda}(x, y)=\int_{0}^{\infty} e^{-z t} \varphi_{z}^{\lambda}(x) \varphi_{z}^{\lambda}(y) d \mu_{\lambda}(z), \quad t, x, y>0,
$$

and the Poisson integral of a function $f$ is defined as

$$
P_{t}^{\lambda} f(x)=\int_{0}^{\infty} P_{t}^{\lambda}(x, y) f(y) d \mu_{\lambda}(y), \quad t, x>0 .
$$

It is noteworthy that also the Poisson kernel can be computed explicitly, and the resulting expression contains the Gauss hypergeometric function ${ }_{2} F_{1}$ (see Section 6 for details).

Exactly the same mapping properties as for $W_{*}^{\lambda}$ turn out to be in force for the Poisson integral maximal operator $P_{*}^{\lambda} f=\sup _{t>0}\left|P_{t}^{\lambda} f\right|$.

Theorem 2.2. Let $\lambda>-1 / 2,1 \leq p<\infty, \delta \in \mathbb{R}$. Then the maximal operator $P_{*}^{\lambda}$, considered on the measure space $\left(\mathbb{R}_{+}, x^{\delta} d x\right)$, has the following mapping properties:

(a) $P_{*}^{\lambda}$ is of strong type $(p, p)$ iff $p>1$ and $-1<\delta<(2 \lambda+1) p-1$;

(b) $P_{*}^{\lambda}$ is of weak type $(p, p)$ iff $-1<\delta<(2 \lambda+1) p-1$ or $\delta=2 \lambda$;

(c) $P_{*}^{\lambda}$ is of restricted weak type $(p, p)$ iff $-1<\delta \leq(2 \lambda+1) p-1$.

Moreover, $P_{*}^{\lambda}$ is of strong type $(\infty, \infty)$. 
We now pass to the Riesz transform. The Bessel operator can be represented as

$$
\Delta_{\lambda}=D^{*} D,
$$

where $D=d / d x$ is the usual derivative and $D^{*}=-x^{-2 \lambda}(d / d x) x^{2 \lambda}$ is the formal adjoint of $D$ in $L^{2}\left(\mathbb{R}_{+}, d \mu_{\lambda}\right)$. This factorization suggests the following system of Cauchy-Riemann type equations:

$$
\frac{\partial}{\partial t} P_{t}^{\lambda} f(x)=-D_{x}^{*} Q_{t}^{\lambda} f(x), \quad \frac{\partial}{\partial t} Q_{t}^{\lambda} f(x)=-D_{x} P_{t}^{\lambda} f(x),
$$

with $Q_{t}^{\lambda} f$ being a suitably defined conjugate Poisson integral,

$$
Q_{t}^{\lambda} f(x)=\int_{0}^{\infty} Q_{t}^{\lambda}(x, y) f(y) d \mu_{\lambda}(y), \quad t, x>0 .
$$

For $\lambda>0$ the conjugate Poisson kernel consistent with the Cauchy-Riemann type equations has the form

$$
Q_{t}^{\lambda}(x, y)=-\frac{2 \lambda}{\pi} \int_{0}^{\pi} \frac{(x-y \cos \theta)(\sin \theta)^{2 \lambda-1}}{\left(x^{2}+y^{2}+t^{2}-2 x y \cos \theta\right)^{\lambda+1}} d \theta, \quad t, x, y>0 .
$$

Then the Riesz transform $R_{\lambda} f$ emerges in a natural way as the boundary value of $Q_{t}^{\lambda} f$,

$$
R_{\lambda} f(x)=\lim _{t \rightarrow 0^{+}} Q_{t}^{\lambda} f(x) .
$$

This is the classical way of defining $R_{\lambda}$ used by Muckenhoupt and Stein [13]. It is known that for each $f \in L^{p}\left(\mathbb{R}_{+}, d \mu_{\lambda}\right), 1 \leq p<\infty$, the above limit exists for almost every $x>0$.

Nevertheless, our approach to the Riesz transform is more direct, and the definition is based on a singular integral representation. By the results of [3, both definitions are consistent when $\lambda>0$.

In agreement with a general philosophy, formally the Riesz transform $R_{\lambda}$ related to $\Delta_{\lambda}$ has the form

$$
R_{\lambda} f=D \Delta_{\lambda}^{-1 / 2} f .
$$

This becomes rigorous provided that $f \in C_{c}^{\infty}\left(\mathbb{R}_{+}\right)$and $\lambda>0$, with the potential operator $\Delta_{\lambda}^{-1 / 2}$ expressed in terms of the Poisson integral,

$$
\Delta_{\lambda}^{-1 / 2} f(x)=\int_{0}^{\infty} P_{t}^{\lambda} f(x) d t, \quad x>0
$$

(see [3]). In the present paper, in contrast with [13, 3, 4], we consider the Riesz transform $R_{\lambda}$ for the full range $\lambda>-1 / 2$. Moreover, to define precisely 
the operator $\Delta_{\lambda}^{-1 / 2}$ we use the heat integral rather than the Poisson one,

$$
\Delta_{\lambda}^{-1 / 2} f(x)=\frac{1}{\sqrt{\pi}} \int_{0}^{\infty}\left(W_{t}^{\lambda} f(x)-\chi_{\{\lambda \leq 0\}} W_{t}^{\lambda} f(0)\right) \frac{d t}{\sqrt{t}}, \quad x>0 .
$$

It will be shown in Section 4 that the limit $W_{t}^{\lambda} f(0)=\lim _{x \rightarrow 0^{+}} W_{t}^{\lambda} f(x)$ exists for each $t>0$ and $\Delta_{\lambda}^{-1 / 2} f(x)$ is well defined for $x>0$, provided that $f \in C_{c}^{\infty}\left(\mathbb{R}_{+}\right)$. Note that for $-1 / 2<\lambda \leq 0$ we have to consider compensated potentials in order to ensure convergence of the defining integral. Then the Riesz transform $R_{\lambda}$ of $f \in C_{c}^{\infty}\left(\mathbb{R}_{+}\right)$is defined by 2.3. Moreover, for $f \in$ $C_{c}^{\infty}\left(\mathbb{R}_{+}\right)$,

$$
R_{\lambda} f(x)=\text { p.v. } \int_{0}^{\infty} R_{\lambda}(x, y) f(y) d \mu_{\lambda}(y), \quad x>0,
$$

with the Riesz transform kernel

$$
R_{\lambda}(x, y)=\frac{1}{\sqrt{\pi}} \int_{0}^{\infty} \frac{\partial}{\partial x} W_{t}^{\lambda}(x, y) \frac{d t}{\sqrt{t}}, \quad x, y>0, x \neq y .
$$

All the details will be given in Section 4. Now we state the boundedness properties of $R_{\lambda}$ (see also Figure 2 below). Notice that $R_{\lambda}$ behaves better than the maximal operators.

Theorem 2.3. Let $\lambda>-1 / 2,1 \leq p<\infty, \delta \in \mathbb{R}$. Then the Riesz transform $R_{\lambda}$, considered on the measure space $\left(\mathbb{R}_{+}, x^{\delta} d x\right)$, has the following mapping properties:

(a) $R_{\lambda}$ is of strong type $(p, p)$ iff $p>1$ and $-1-p<\delta<(2 \lambda+1) p-1$;

(b) $R_{\lambda}$ is weak type $(p, p)$ iff $-1-p<\delta<(2 \lambda+1) p-1$ or $\delta \in\{-2,2 \lambda\}$;

(c) $R_{\lambda}$ is of restricted weak type $(p, p)$ iff $-1-p \leq \delta \leq(2 \lambda+1) p-1$.

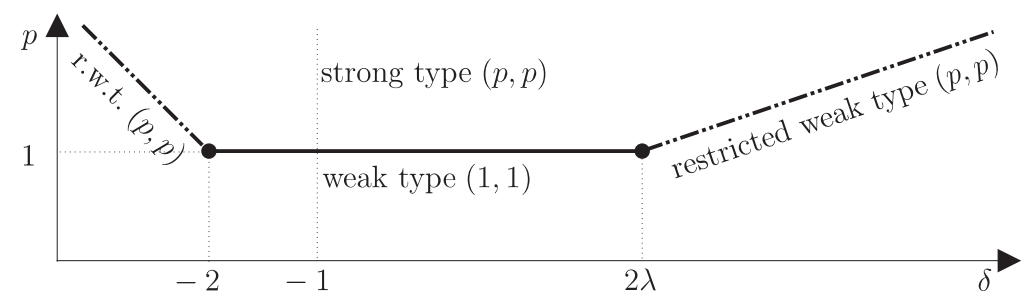

Fig. 2. Mapping properties of $R_{\lambda}($ fixed $\lambda=1)$

We now briefly comment on the adjoint Riesz transform $R_{\lambda}^{*}$. This operator is intimately connected with $R_{\lambda}$ (see for instance the identity 2.5) below). In [13, (16.8)] it was shown that for $\lambda>0$,

$$
R_{\lambda} f=-x h_{\lambda+1 / 2}\left(y^{-1} h_{\lambda-1 / 2}(f)\right), \quad f \in L^{2}\left(\mathbb{R}_{+}, d \mu_{\lambda}\right),
$$


where $h_{\nu}, \nu>-1$, denotes the modified Hankel transform,

$$
\begin{aligned}
h_{\nu}(f)(x) & =\int_{0}^{\infty} \varphi_{x}^{\nu+1 / 2}(y) f(y) d \mu_{\nu+1 / 2}(y) \\
& =\int_{0}^{\infty}(x y)^{-\nu} J_{\nu}(x y) f(y) y^{2 \nu+1} d y, \quad x>0 .
\end{aligned}
$$

It can be proved that (2.4) holds in fact for all $\lambda>-1 / 2$. Since $h_{\nu}$ is an isometry in $L^{2}\left(\mathbb{R}_{+}, d \mu_{\lambda}\right)$ and the Parseval type identity

$$
\int_{0}^{\infty} h_{\nu}(f)(x) g(x) x^{2 \nu+1} d x=\int_{0}^{\infty} f(x) h_{\nu}(g)(x) x^{2 \nu+1} d x,
$$

$f, g \in L^{2}\left(\mathbb{R}_{+}, d \mu_{\lambda}\right)$, holds for every $\nu>-1$ (cf. [5]), the adjoint of $R_{\lambda}$ is given by

$$
R_{\lambda}^{*} f=-h_{\lambda-1 / 2}\left(y h_{\lambda+1 / 2}\left(x^{-1} f\right)\right), \quad f \in L^{2}\left(\mathbb{R}_{+}, d \mu_{\lambda}\right) .
$$

Further, since $h_{\nu}^{-1}=h_{\nu}$ in $L^{2}\left(\mathbb{R}_{+}, d \mu_{\lambda}\right)$ for all $\nu>-1$, it becomes clear that

$$
R_{\lambda}^{*} R_{\lambda} f=R_{\lambda} R_{\lambda}^{*} f=f, \quad f \in L^{2}\left(\mathbb{R}_{+}, d \mu_{\lambda}\right), \lambda>-1 / 2 .
$$

On the other hand, it is remarkable that $R_{\lambda}^{*}$ emerges as the Riesz transform naturally associated with the Bessel type operator

$$
D D^{*}=\Delta_{\lambda}+\frac{2 \lambda}{x^{2}}=-\frac{d^{2}}{d x^{2}}-\frac{2 \lambda}{x} \frac{d}{d x}+\frac{2 \lambda}{x^{2}} .
$$

Namely, formally we have $R_{\lambda}^{*}=D^{*}\left(D D^{*}\right)^{-1 / 2}$. This identity can be given a precise meaning, but we shall not go into details here to avoid confusion with the line of thought of the paper.

The results concerning $R_{\lambda}^{*}$ can be summarized as follows.

Proposition 2.4. Let $\lambda>-1 / 2,1 \leq p<\infty, \delta \in \mathbb{R}$. Then the adjoint Riesz transform $R_{\lambda}^{*}$, considered on the measure space $\left(\mathbb{R}_{+}, x^{\delta} d x\right)$, has the following mapping properties:

(a) $R_{\lambda}^{*}$ is of strong type $(p, p)$ iff $p>1$ and $-1<\delta<2(\lambda+1) p-1$;

(b) $R_{\lambda}^{*}$ is of weak type $(p, p)$ iff $-1<\delta<2(\lambda+1) p-1$ or $\delta=2 \lambda+1$;

(c) $R_{\lambda}^{*}$ is of restricted weak type $(p, p)$ iff $-1<\delta \leq 2(\lambda+1) p-1$.

Moreover,

$$
R_{\lambda}^{*} R_{\lambda} f=R_{\lambda} R_{\lambda}^{*} f=f, \quad f \in L^{p}\left(\mathbb{R}_{+}, x^{\delta} d x\right),
$$

provided that $p>1$ and $-1<\delta<(2 \lambda+1) p-1$.

Observe that (a) above can be directly deduced from the strong type result for the Riesz transform. For the other items we may apply the same arguments as for $R_{\lambda}$ because, as we shall see, they rely on pointwise estimates 
of the kernel $R_{\lambda}(x, y)$. Since the kernel of $R_{\lambda}^{*}$ is $R_{\lambda}(y, x)$, we easily obtain the same kind of estimates for the adjoint Riesz transform.

Finally, consider the Littlewood-Paley type square function

$$
g_{\lambda}(f)(x)=\left(\int_{0}^{\infty} t\left|\frac{\partial}{\partial t} W_{t}^{\lambda} f(x)\right|^{2} d t\right)^{1 / 2} .
$$

We prove the following boundedness properties of $g_{\lambda}$.

Theorem 2.5. Let $\lambda>-1 / 2,1 \leq p<\infty, \delta \in \mathbb{R}$. Then the square function $g_{\lambda}$, considered on the measure space $\left(\mathbb{R}_{+}, x^{\delta} d x\right)$, has the following mapping properties:

(a) $g_{\lambda}$ is of strong type $(p, p)$ iff $p>1$ and $-1<\delta<(2 \lambda+1) p-1$;

(b) $g_{\lambda}$ is of weak type $(p, p)$ iff $-1<\delta<(2 \lambda+1) p-1$ or $\delta=2 \lambda$;

(c) $g_{\lambda}$ is of restricted weak type $(p, p)$ iff $-1<\delta \leq(2 \lambda+1) p-1$.

Notice that the behavior of $g_{\lambda}$ is exactly the same as that of the maximal operators $W_{*}^{\lambda}$ and $P_{*}^{\lambda}$ (see Figure 1). Note also that the Riesz transform and its adjoint, taken into account individually, behave better than the maximal operators. However, if considered simultaneously, they lead back to precisely the same mapping properties as those of $W_{*}^{\lambda}$ and $P_{*}^{\lambda}$. In addition, it is worth mentioning that the maximal operators are bounded on $L^{p}\left(\mathbb{R}_{+}, x^{\delta} d \mu_{\lambda}\right)$ for given $1<p<\infty$ and $\lambda>-1 / 2$ if and only if $x^{\delta} \in A_{p}^{\lambda}$; here $A_{p}^{\lambda}=$ $A_{p}\left(\mathbb{R}_{+}, d \mu_{\lambda}\right)$ denotes the Muckenhoupt class of $A_{p}$ weights associated with the homogeneous space $\left(\mathbb{R}_{+},|\cdot|, d \mu_{\lambda}\right)$.

As was already indicated in the Introduction, several harmonic analysis operators associated with the Bessel operator

$$
\widetilde{\Delta}_{\lambda}=x^{\lambda} \Delta_{\lambda} x^{-\lambda}=-x^{-\lambda} D x^{2 \lambda} D x^{-\lambda}=\widetilde{D}^{*} \widetilde{D}, \quad \widetilde{D}=x^{\lambda} D x^{-\lambda},
$$

were studied earlier, usually with the assumption $\lambda>0$. We now explain how our present results are related to those in [3, 4]. Recall that $\widetilde{\Delta}_{\lambda}$ is associated with the Lebesgue measure space $\left(\mathbb{R}_{+}, d x\right)$. Consider the multiplication operator $V_{\lambda} f(x)=x^{-\lambda} f(x)$, which is obviously an isometry between $L^{2}\left(\mathbb{R}_{+}, d x\right)$ and $L^{2}\left(\mathbb{R}_{+}, d \mu_{\lambda}\right)$. An essential observation is that $V_{\lambda}$ intertwines all relevant operators in both settings in question and also establishes an $L^{2}$ equivalence between them. More precisely, distinguishing with tildes appropriately defined objects in the $\widetilde{\Delta}_{\lambda}$ setting, we have $\widetilde{\Delta}_{\lambda}=V_{-\lambda} \Delta_{\lambda} V_{\lambda}, \widetilde{D}=V_{-\lambda} D V_{\lambda}$, $\widetilde{W}_{*}^{\lambda}=V_{-\lambda} W_{*}^{\lambda} V_{\lambda}, \widetilde{P}_{*}^{\lambda}=V_{-\lambda} P_{*}^{\lambda} V_{\lambda}, \widetilde{R}_{\lambda}=V_{-\lambda} R_{\lambda} V_{\lambda}, \widetilde{g}_{\lambda}=V_{-\lambda} g_{\lambda} V_{\lambda}$. Consequently, we can deduce from Theorems 2.1 2.5 the strong boundedness results in $L^{p}\left(\mathbb{R}_{+}, x^{\delta} d x\right)$ for the corresponding operators in the $\widetilde{\Delta}_{\lambda}$ context. Furthermore, by applying the same procedures as in the proofs of the theorems just mentioned, one can also obtain the desired weak type and restricted weak type mapping properties, as well as their sharpness. These results, appropriately stated and justified, complement those from [3, 4]. After proving 
Theorems 2.1, 2.3 and 2.5 we provide remarks concerning the boundedness properties of the operators associated with $\widetilde{\Delta}_{\lambda}$.

An important ingredient of the proofs contained in the following sections are basic differential and asymptotic properties of the modified Bessel function $I_{\nu}$. Those listed below can be found for instance in [18] or [12].

One of possible definitions of $I_{\nu}(z)$ for, say, $\nu>-1$ and $z>0$ is

$$
I_{\nu}(z)=\sum_{n=0}^{\infty} \frac{(z / 2)^{2 n+\nu}}{\Gamma(n+1) \Gamma(n+\nu+1)} .
$$

A straightforward analysis of the above series shows that

$$
\frac{d}{d z}\left(z^{-\nu} I_{\nu}(z)\right)=z^{-\nu} I_{\nu+1}(z), \quad z>0, \nu>-1,
$$

and

$$
\lim _{z \rightarrow 0^{+}} z^{-\nu} I_{\nu}(z)=\frac{1}{2^{\nu} \Gamma(\nu+1)}, \quad \nu>-1 .
$$

Furthermore, for $z>0$ and $\nu>-1$, we have the following asymptotic representation: given $n=0,1,2, \ldots$,

$$
I_{\nu}(z)=\frac{e^{z}}{\sqrt{2 \pi z}}\left(\sum_{k=0}^{n}(-1)^{k}[\nu, k](2 z)^{-k}+\mathcal{O}\left(z^{-n-1}\right)\right),
$$

the coefficients being specified by $[\nu, 0]=1$ and

$$
[\nu, k]=\frac{\left(4 \nu^{2}-1\right)\left(4 \nu^{2}-3^{2}\right) \cdot \ldots \cdot\left(4 \nu^{2}-(2 k-1)^{2}\right)}{2^{2 k} \Gamma(k+1)}, \quad k=1,2, \ldots
$$

Objects that will frequently appear in our estimates are the Hardy type operators

$$
\begin{aligned}
H_{0}^{\eta} f(x) & =x^{-\eta-1} \int_{0}^{x} f(y) y^{\eta} d y, \quad x>0, \\
H_{\infty}^{\eta} f(x) & =x^{\eta} \int_{x}^{\infty} f(y) y^{-\eta-1} d y, \quad x>0,
\end{aligned}
$$

considered for $\eta>-1$. The relevant mapping properties of $H_{0}^{\eta}$ and $H_{\infty}^{\eta}$ are gathered in [8, Lemmas 3.1 and 3.2] or [9, Lemmas 3 and 4] (see also references given there). For the sake of completeness and reader's convenience, we reproduce them below.

Lemma 2.6. Let $\eta>-1$ and consider $H_{0}^{\eta}$ on the measure space $\left(\mathbb{R}_{+}, x^{\delta} d x\right)$.

(a) $H_{0}^{\eta}$ is of strong type $(p, p)$ when $1<p \leq \infty$ and $\delta<p(\eta+1)-1$;

(b) $H_{0}^{\eta}$ is of weak type $(1,1)$ if $\delta \leq \eta$;

(c) $H_{0}^{\eta}$ is of restricted weak type $(p, p)$ if $1<p<\infty$ and $\delta=p(\eta+1)-1$. 
Lemma 2.7. Let $\eta>-1$ and consider $H_{\infty}^{\eta}$ on the measure space $\left(\mathbb{R}_{+}, x^{\delta} d x\right)$.

(a) $H_{\infty}^{\eta}$ is of strong type $(p, p)$ when $1<p<\infty$ and $-\eta p-1<\delta$;

(b) $H_{\infty}^{\eta}$ is of strong type $(\infty, \infty)$ for any $\delta \in \mathbb{R}$ if only $\eta>0$;

(c) $H_{\infty}^{\eta}$ is of weak type $(1,1)$ when $-\eta-1 \leq \delta(<$ if $\eta=0)$;

(d) $H_{\infty}^{\eta}, \eta \neq 0$, is of restricted weak type $(p, p)$ when $1<p<\infty$ and $\delta=-\eta p-1$.

Another object that will be used throughout is the Gauss-Weierstrass kernel

$$
\mathcal{W}_{t}(x, y)=\frac{1}{\sqrt{4 \pi t}} e^{-(x-y)^{2} / 4 t}, \quad t>0, x, y \in \mathbb{R} .
$$

The associated heat integral,

$$
\mathcal{W}_{t} f(x)=\int_{\mathbb{R}} \mathcal{W}_{t}(x, y) f(y) d y, \quad x \in \mathbb{R},
$$

represents the classical heat semigroup $\left\{\mathcal{W}_{t}\right\}_{t>0}$ on the real line. A crucial argument used repeatedly below relies on a comparison of various operators in the Bessel setting with the corresponding well-known operators related to $\mathcal{W}_{t}$.

To establish weighted $L^{p}$ mapping properties of certain auxiliary operators appearing in the proofs of Theorems 2.3 and 2.5. we shall use the following result that can be proved by applying the local version of the Calderón-Zygmund operator theory on the real line (or rather its vectorvalued variant), developed recently by Nowak and Stempak [14, Section 4]. It is remarkable that the results from [14] remain valid in a vector-valued setting.

Lemma 2.8. Let $(B,\|\cdot\|)$ be a separable Banach space. Assume that $T$ is a local vector-valued Calderón-Zygmund operator, i.e. $T$ is a bounded operator from $L^{2}\left(\mathbb{R}_{+}, d x\right)$ into the Lebesgue-Bochner space $L_{B}^{2}\left(\mathbb{R}_{+}, d x\right)$ such that

$$
T f(x)=\int_{x / 2}^{2 x} K(x, y) f(y) d y, \quad \text { a.e. } x \notin \operatorname{supp} f, f \in C_{c}^{\infty}\left(\mathbb{R}_{+}\right),
$$

where the B-valued kernel is weakly measurable and satisfies the standard estimates

$$
\|K(x, y)\| \leq \frac{C}{|x-y|}, \quad\left\|\nabla_{x, y} K(x, y)\right\| \leq \frac{C}{|x-y|^{2}},
$$

in the "local" region $0<x / 2<y<2 x, x \neq y$. Then, for each $\lambda \in \mathbb{R}$, the operator $S_{\lambda}$ defined by

$$
S_{\lambda} f(x)=x^{-\lambda} T\left(y^{\lambda} f\right)(x), \quad f \in C_{c}^{\infty}\left(\mathbb{R}_{+}\right),
$$


is also a local vector-valued Calderón-Zygmund operator and consequently, given any $\delta \in \mathbb{R}$, it extends to a bounded operator from $L^{p}\left(\mathbb{R}_{+}, x^{\delta} d x\right)$ into $L_{B}^{p}\left(\mathbb{R}_{+}, x^{\delta} d x\right), 1<p<\infty$, and from $L^{1}\left(\mathbb{R}_{+}, x^{\delta} d x\right)$ into $L_{B}^{1, \infty}\left(\mathbb{R}_{+}, x^{\delta} d x\right)$.

This lemma can be justified, in a straightforward manner, by applying a vector-valued variant of [14, Theorem 4.3] and using the fact that each $A_{\text {loc }}^{p}$ class (considered in [14]), $1 \leq p<\infty$, contains all power weights $x^{\delta}, \delta \in \mathbb{R}$.

Throughout the paper we use the convention that constants may change their value (but not the dependence) from one occurrence to the next. The notation $c_{p}$ means that the constant depends only on $p$. Constants are always strictly positive and finite. Moreover, we distinguish "large" and "small" constants by using capital and small letters, respectively.

Finally, we shall implicitly use the simple fact that $\sup _{t>0} t^{\beta} \exp (-\gamma t)=$ $C_{\beta, \gamma}<\infty$ for arbitrary $\beta, \gamma>0$.

3. The heat integral maximal operator. In this section we prove Theorem 2.1. Recall that for $\lambda>-1 / 2$,

$$
W_{*}^{\lambda} f(x)=\sup _{t>0}\left|\int_{0}^{\infty} W_{t}^{\lambda}(x, y) f(y) d \mu_{\lambda}(y)\right|, \quad x>0,
$$

with the heat kernel given by 2.2. We shall use the following estimates of $W_{t}^{\lambda}(x, y)$.

Lemma 3.1. Let $\lambda>-1 / 2$. Then for all $t, x, y>0$,

$$
W_{t}^{\lambda}(x, y) \leq C_{\lambda} \begin{cases}x^{-2 \lambda-1}, & y \leq x / 2, \\ x^{-2 \lambda-1}+t^{-1 / 2}(x y)^{-\lambda} e^{-(x-y)^{2} / 4 t}, & x / 2<y<2 x, \\ y^{-2 \lambda-1}\left(y^{2} / t\right)^{\lambda+1 / 2} e^{-c y^{2} / t}, & 2 x \leq y .\end{cases}
$$

Proof. First observe that if $x y \leq t$ then $(2.8)$ implies

$$
W_{t}^{\lambda}(x, y) \leq C_{\lambda} \frac{1}{t^{\lambda+1 / 2}} e^{-\left(x^{2}+y^{2}\right) / 4 t} \leq C_{\lambda} \frac{1}{y^{2 \lambda+1}}\left(\frac{y^{2}}{t}\right)^{\lambda+1 / 2} e^{-y^{2} / 4 t} .
$$

On the other hand, if $x y>t$ then $(2.9)$ leads to

$$
W_{t}^{\lambda}(x, y) \leq C_{\lambda} \frac{1}{(x y)^{\lambda} \sqrt{t}} e^{-(x-y)^{2} / 4 t} .
$$

Assume now that $2 x \leq y$. Then, in view of (3.1), it is enough to consider $x y>t$ and by 3.2 we get

$$
\begin{aligned}
W_{t}^{\lambda}(x, y) & \leq \frac{C_{\lambda}}{(x y)^{\lambda+1 / 2}}\left(\frac{x y}{t}\right)^{1 / 2} e^{-y^{2} / 16 t} \\
& \leq \frac{C_{\lambda}}{t^{\lambda+1 / 2}}\left(\frac{y^{2}}{t}\right)^{1 / 2} e^{-y^{2} / 16 t}=\frac{C_{\lambda}}{y^{2 \lambda+1}}\left(\frac{y^{2}}{t}\right)^{\lambda+1} e^{-y^{2} / 16 t} .
\end{aligned}
$$

This implies the desired bound for $W_{t}^{\lambda}(x, y)$. 
The case $y \leq x / 2$, by the symmetry $W_{t}^{\lambda}(x, y)=W_{t}^{\lambda}(y, x)$, is easily covered by a direct weakening of the already justified estimate for $2 x \leq y$. Finally, the remaining estimate for comparable $x$ and $y$ follows by combining (3.1) and 3.2.

Consider the auxiliary maximal operator

$$
T f(x)=\sup _{t>0}\left|\int_{x}^{\infty}\left(\frac{y^{2}}{t}\right)^{\lambda+1 / 2} \exp \left(-c \frac{y^{2}}{t}\right) \frac{f(y)}{y} d y\right|, \quad x>0 .
$$

Observe that $T$ can be controlled, up to a multiplicative constant, by the Hardy operator $H_{\infty}^{0}$. Thus $T$ has all the mapping properties stated in Lemma 2.7 with $\eta=0$. Moreover, a straightforward computation shows that $T$ is bounded on $L^{\infty}\left(\mathbb{R}_{+}\right)$. Further results concerning this and the more general operator

$$
T_{\psi}^{\eta} f(x)=\sup _{s>0}\left|x^{\eta} \int_{x}^{\infty} f(y) \psi(s, y) y^{-\eta-1} d y\right|, \quad x>0,
$$

can be found in [8, Lemma 3.3], but they will not be used here except for Remark 3.2 below.

We also invoke the local maximal function $M_{\mathrm{loc}}^{k}$ defined by

$$
M_{\mathrm{loc}}^{k} f(x)=\sup _{0<u<x<v<k u} \frac{1}{v-u} \int_{u}^{v}|f(y)| d y, \quad x>0,
$$

for a given $k>1$. This operator, for any $\delta \in \mathbb{R}$, is bounded on $L^{p}\left(\mathbb{R}_{+}, x^{\delta} d x\right)$, $1<p \leq \infty$, and from $L^{1}\left(\mathbb{R}_{+}, x^{\delta} d x\right)$ to $L^{1, \infty}\left(\mathbb{R}_{+}, x^{\delta} d x\right)$ (see [14, Section 6]).

Proof of Theorem 2.1. In order to show sufficiency parts in Theorem 2.1 we split the kernel $W_{t}^{\lambda}(x, y)$ according to the regions $0<y \leq x / 2$, $x / 2<y<2 x, 2 x \leq y$, and denote the resulting maximal operators by $N_{1}^{\lambda}$, $N_{2}^{\lambda}$ and $N_{3}^{\lambda}$, respectively. Then

$$
W_{*}^{\lambda} f(x) \leq N_{1}^{\lambda}|f|(x)+N_{2}^{\lambda}|f|(x)+N_{3}^{\lambda}|f|(x), \quad x>0 .
$$

Using Lemma 3.1 we get

$$
N_{1}^{\lambda}|f|(x) \leq C_{\lambda} H_{0}^{2 \lambda}|f|(x), \quad x>0 .
$$

Another application of Lemma 3.1 gives

$$
N_{3}^{\lambda}|f|(x) \leq C_{\lambda} T|f|(x), \quad x>0 .
$$

Considering $N_{2}^{\lambda}$, again by Lemma 3.1 we have, for $x>0$,

$$
\begin{aligned}
N_{2}^{\lambda}|f|(x) & \leq C_{\lambda}\left(\int_{x / 2}^{2 x}|f(y)| \frac{d y}{y}+\sup _{t>0} \int_{x / 2}^{2 x} \frac{1}{\sqrt{t}} e^{-(x-y)^{2} / 4 t}|f(y)| d y\right) \\
& \leq C_{\lambda} M_{\mathrm{loc}}^{4} f(x) .
\end{aligned}
$$


Now, taking into account the above estimates and facts, and appealing to Lemmas 2.6 and 2.7, we conclude the following mapping properties of the operator $W_{*}^{\lambda}$, considered on the space $\left(\mathbb{R}_{+}, x^{\delta} d x\right)$. For $1<p<\infty, W_{*}^{\lambda}$ is of strong type $(p, p)$ provided that $-1<\delta<(2 \lambda+1) p-1$. Moreover, $W_{*}^{\lambda}$ is of strong type $(\infty, \infty)$ for each $\delta \in \mathbb{R}$. If $-1<\delta \leq 2 \lambda$ then $W_{*}^{\lambda}$ is of weak type $(1,1)$. Finally, if $1<p<\infty$ and $-1<\delta \leq(2 \lambda+1) p-1$ then $W_{*}^{\lambda}$ is of restricted weak type $(p, p)$. These facts, altogether, justify the sufficiency parts in Theorem 2.1.

We pass to the proof of the necessity parts, that is, showing the sharpness of the above results. Our task will be done once we establish the following three statements (as before, we assume that $\lambda>-1 / 2,1 \leq p<\infty$ and the underlying space is $\left.\left(\mathbb{R}_{+}, x^{\delta} d x\right)\right)$.

(A) If $W_{*}^{\lambda}$ is of restricted weak type $(p, p)$ then $-1<\delta \leq(2 \lambda+1) p-1$.

(B) $W_{*}^{\lambda}$ is not of weak type $(p, p)$ when $p>1$ and $\delta=(2 \lambda+1) p-1$.

(C) $W_{*}^{\lambda}$ is not of strong type $(1,1)$ if $-1<\delta \leq 2 \lambda$.

To this end, let $f$ be a nonnegative function on $(0, \infty)$. Since by 2.8$)$,

$$
W_{t}^{\lambda}(x, y) \geq \frac{c_{\lambda}}{t^{\lambda+1 / 2}} e^{-\left(x^{2}+y^{2}\right) / 4 t}, \quad x y<t,
$$

we see that

$$
W_{t}^{\lambda} f(x) \geq \frac{c_{\lambda}}{t^{\lambda+1 / 2}} \int_{0}^{t / x} e^{-\left(x^{2}+y^{2}\right) / 4 t} f(y) d \mu_{\lambda}(y), \quad t, x>0 .
$$

Consequently, since $W_{*}^{\lambda} f(x) \geq W_{x^{2}}^{\lambda} f(x), x>0$, we get

$$
W_{*}^{\lambda} f(x) \geq \frac{c_{\lambda}}{x^{2 \lambda+1}} \int_{0}^{x} e^{-\left(x^{2}+y^{2}\right) / 4 x^{2}} f(y) d \mu_{\lambda}(y) \geq \frac{c_{\lambda}}{x^{2 \lambda+1}} \int_{0}^{x} f(y) d \mu_{\lambda}(y) .
$$

Similarly, by 2.9 we have

$$
W_{t}^{\lambda} f(x) \geq \frac{c_{\lambda}}{\sqrt{t}} \int_{t / x}^{\infty} \frac{1}{(x y)^{\lambda}} e^{-(x-y)^{2} / 4 t} f(y) d \mu_{\lambda}(y), \quad t, x>0,
$$

and therefore

$$
W_{*}^{\lambda} f(x) \geq \frac{c_{\lambda}}{x} \int_{x}^{\infty} \frac{1}{(x y)^{\lambda}} e^{-(x-y)^{2} / 4 x^{2}} f(y) d \mu_{\lambda}(y), \quad x>0 .
$$

Suppose now that $1 \leq p<\infty$ and $W_{*}^{\lambda}$ can be extended from $L^{2}\left(\mathbb{R}_{+}, d \mu_{\lambda}\right)$ to a restricted weak type $(p, p)$ operator on $\left(\mathbb{R}_{+}, x^{\delta} d x\right)$. Considering $f=$ $\chi_{(1,2)}$, by 3.3 we obtain

$$
W_{*}^{\lambda} f(x) \geq c_{\lambda} x^{-2 \lambda-1}, \quad x \geq 2 .
$$


Then, by the weak type $(p, p)$ inequality satisfied by $f$,

$$
\int_{2}^{\gamma^{-1 /(2 \lambda+1)}} y^{\delta} d y \leq C_{p, \lambda} \gamma^{-p}
$$

for $\gamma>0$ sufficiently small. It follows that the function $\gamma \mapsto \gamma^{p-(\delta+1) /(2 \lambda+1)}$ must be bounded for $\gamma$ near 0 and we conclude that $\delta \leq(2 \lambda+1) p-1$. On the other hand, in view of $(3.5)$, for $f=\chi_{(1,2)}$ as above and $x \in(0,1)$,

$$
W_{*}^{\lambda} f(x) \geq \frac{c_{\lambda}}{x^{\lambda+1}} \int_{1}^{2} y^{\lambda} e^{-(x-y)^{2} / 4 x^{2}} d y \geq \frac{c_{\lambda}}{x^{\lambda+1}},
$$

which together with the weak type $(p, p)$ inequality for $f$ implies $\int_{0}^{1} x^{\delta} d x<\infty$. The conclusion $\delta>-1$ follows and this completes proving statement (A).

Next, fix $1<p<\infty$, let $\delta=(2 \lambda+1) p-1$ and suppose, on the contrary, that $W_{*}^{\lambda}$ can be extended to a weak type $(p, p)$ operator on $\left(\mathbb{R}_{+}, x^{\delta} d x\right)$. It is straightforward that with $p^{\prime}$ being the conjugate of $p, 1 / p+1 / p^{\prime}=1$, the integral $\int_{0}^{1} x^{(2 \lambda-\delta) p^{\prime}+\delta} d x$ is infinite. Therefore, for each $n \geq 1$ there exists a nonnegative function $f_{n} \in L^{2}\left((0,1), d \mu_{\lambda}\right) \cap L^{p}\left((0,1), x^{\delta} d x\right)$ such that $\left\|f_{n}\right\|_{L^{p}\left((0,1), x^{\delta} d x\right)} \leq 1$ and $\int_{0}^{1} f_{n}(x) d \mu_{\lambda}(x) \geq n$. Extending $f_{n}$ to $\mathbb{R}_{+}$by letting $f_{n}(x)=0$ for $x \geq 1$ and making use of (3.3) we can write

$$
W_{*}^{\lambda} f_{n}(x) \geq c_{\lambda} n x^{-2 \lambda-1}, \quad x \geq 1, n=1,2, \ldots
$$

Now, in view of the weak type $(p, p)$ boundedness of $W_{*}^{\lambda}$, we get

$$
\int_{1}^{(n /(n-1))^{1 /(2 \lambda+1)}} y^{\delta} d y \leq C_{\lambda}\left(\frac{\left\|f_{n}\right\|_{L^{p}\left((0,1), x^{\delta} d x\right)}}{n-1}\right)^{p} \leq C_{\lambda} \frac{1}{(n-1)^{p}}
$$

for $n=2,3, \ldots$. This in turn implies the boundedness of the sequence $\left\{n^{p}-(n-1)^{p}: n \geq 2\right\}$, a contradiction because $p>1$. Thus statement (B) is justified.

It remains to prove that $W_{*}^{\lambda}$ is not strong type $(1,1)$ on $\left(\mathbb{R}_{+}, x^{\delta} d x\right)$ when $-1<\delta \leq 2 \lambda$. Assuming that $x \geq 1$ and $t \leq 1 / 2$ and restricting the interval of integration in (3.4) we get

$$
\begin{aligned}
W_{t}^{\lambda} f(x) & \geq c_{\lambda} \int_{x / 2}^{2 x} \frac{1}{\sqrt{t}(x y)^{\lambda}} e^{-(x-y)^{2} / 4 t} f(y) d \mu_{\lambda}(y) \\
& \geq c_{\lambda} \frac{1}{\sqrt{t}} \int_{x / 2}^{2 x} e^{-(x-y)^{2} / 4 t} f(y) d y, \quad x \geq 1, t \leq 1 / 2 .
\end{aligned}
$$

For $0<\varepsilon<1 / 2$ consider $1<y<1+\varepsilon$ and $1+2 \varepsilon<x<2$. Then obviously $x / 2<y<2 x$ and, moreover, if $t=(x-1)^{2} / 2$ (notice that this quantity is 
less than $1 / 2$ ) we have

$$
\frac{(x-y)^{2}}{4 t} \leq \frac{1}{2}\left(\frac{x-1+y-1}{x-1}\right)^{2} \leq \frac{1}{2}\left(1+\frac{\varepsilon}{2 \varepsilon}\right)^{2}=\frac{9}{8} .
$$

Therefore, choosing $f_{\varepsilon}=\chi_{(1,1+\varepsilon)}$, we arrive at

$$
W_{*}^{\lambda} f_{\varepsilon}(x) \geq c_{\lambda} \frac{\varepsilon}{x-1}, \quad 1+2 \varepsilon<x<2 .
$$

Consequently,

$$
\int_{0}^{\infty}\left|W_{*}^{\lambda} f_{\varepsilon}(x)\right| x^{\delta} d x \geq c_{\lambda} \varepsilon \int_{1+2 \varepsilon}^{2} \frac{x^{\delta}}{x-1} d x \geq c_{\lambda, \delta} \varepsilon \int_{1+2 \varepsilon}^{2} \frac{d x}{x-1}=c_{\lambda, \delta} \varepsilon \log \frac{1}{2 \varepsilon} .
$$

On the other hand, clearly $\left\|f_{\varepsilon}\right\|_{L^{1}\left(\mathbb{R}_{+}, x^{\delta} d x\right)} \leq C_{\delta} \varepsilon$. Letting $\varepsilon \rightarrow 0^{+}$we see that $W_{*}^{\lambda}$ is not bounded on $L^{1}\left(\mathbb{R}_{+}, x^{\delta} d x\right)$. Statement $(\mathrm{C})$ follows.

The proof of Theorem 2.1 is now complete.

REMARK 3.2. The boundedness properties of the maximal operator $\widetilde{W}_{*}^{\lambda}$ can be obtained by following the proof of Theorem 2.1. taking into account that $\widetilde{W}_{t}^{\lambda}(x, y)=(x y)^{\lambda} W_{t}^{\lambda}(x, y)$. In fact, Lemma 3.1 implies that $\widetilde{W}_{*}^{\lambda} f$ can be controlled by $H_{0}^{\lambda} f+M_{\mathrm{loc}}^{4} f+T_{\psi}^{\lambda} f$, with $\psi(s, y)=\left(y^{2} / s\right)^{\lambda+1 / 2} e^{-c y^{2} / s}$. Invoking the mapping properties of $T_{\psi}^{\lambda}$ from [8, Lemma 3.3], and making use of (3.3) and (3.5), leads to similar results for $\widetilde{W}_{*}^{\lambda}$ to those stated in Theorem 2.1, just replacing the interval $(-1,(2 \lambda+1) p-1)$ by $(-\lambda p-1,(\lambda+1) p-1)$. However, in contrast with the $\Delta_{\lambda}$ setting, these parallel results are not uniform in $\lambda$. A singularity occurs at $\lambda=0$, exactly as described by the statements of $[8$, Theorem 2.2] with $\alpha$ replaced by $\lambda-1 / 2$.

4. Riesz transform. In this section we prove Theorem 2.3. Recall that the Riesz transform associated with the Bessel operator $\Delta_{\lambda}$ is formally defined by

$$
R_{\lambda}=D \Delta_{\lambda}^{-1 / 2}, \quad \lambda>-1 / 2 .
$$

We begin with a rigorous definition of the operator $\Delta_{\lambda}^{-1 / 2}$. Then we obtain a representation of $R_{\lambda}$ in terms of a principal value integral.

The negative power of $\Delta_{\lambda}$ can be defined, at least for smooth functions with compact support, by

$$
\Delta_{\lambda}^{-1 / 2} f(x)=\frac{1}{\sqrt{\pi}} \int_{0}^{\infty}\left(W_{t}^{\lambda} f(x)-\chi_{\{\lambda \leq 0\}} W_{t}^{\lambda} f(0)\right) \frac{d t}{\sqrt{t}},
$$

with $W_{t}^{\lambda} f(0)$ understood as $\lim _{x \rightarrow 0^{+}} W_{t}^{\lambda} f(x)$. Note that the compensating term is necessary to make the integral convergent when $-1 / 2<\lambda \leq 0$, as can be seen below. 
Proposition 4.1. Let $\lambda>-1 / 2$ and $f \in C_{c}^{\infty}\left(\mathbb{R}_{+}\right)$. Then the limit $W_{t}^{\lambda} f(0)$ exists for each fixed $t>0$, and the function $\Delta_{\lambda}^{-1 / 2} f(x), x>0$, is well defined.

Proof. Suppose that $\operatorname{supp} f \subset(a, b), 0<a<b<\infty$. To see that the limit exists it is sufficient to notice that, in view of (2.8), we have

$$
W_{t}^{\lambda}(0, y):=\lim _{x \rightarrow 0^{+}} W_{t}^{\lambda}(x, y)=\frac{1}{2^{2 \lambda} \Gamma(\lambda+1 / 2)} \frac{1}{t^{\lambda+1 / 2}} e^{-y^{2} / 4 t},
$$

the convergence being uniform in $y<b$.

Now fix $x>0$. By using (2.6) we get

$$
\begin{aligned}
& \int_{1}^{\infty}\left|W_{t}^{\lambda} f(x)-\chi_{\{\lambda \leq 0\}} W_{t}^{\lambda} f(0)\right| \frac{d t}{\sqrt{t}} \\
& \leq \int_{1}^{\infty} \int_{a}^{b} \mid \frac{(x y)^{-\lambda+1 / 2}}{2 t} I_{\lambda-1 / 2}\left(\frac{x y}{2 t}\right) e^{-\left(x^{2}+y^{2}\right) / 4 t} \\
& \quad-\frac{\chi_{\{\lambda \leq 0\}} e^{-y^{2} / 4 t}}{t^{\lambda+1 / 2} 2^{2 \lambda} \Gamma(\lambda+1 / 2)}|f(y)| d \mu_{\lambda}(y) \frac{d t}{\sqrt{t}} \\
& \leq C_{\lambda, b, x} \int_{1}^{\infty} \int_{a}^{b}\left(\left|e^{-\left(x^{2}+y^{2}\right) / 4 t}-\chi_{\{\lambda \leq 0\}} e^{-y^{2} / 4 t}\right|+\frac{(x y)^{2}}{t^{2}}\right)|f(y)| d \mu_{\lambda}(y) \frac{d t}{t^{\lambda+1}} \\
& \leq C_{\lambda, b, x} \int_{a}^{\infty} \int_{1}^{\infty}\left(\frac{\chi_{\{\lambda \leq 0\}} x^{2}}{t^{\lambda+2}}+\frac{\chi_{\{\lambda>0\}}}{t^{\lambda+1}}+\frac{(x y)^{2}}{t^{\lambda+3}}\right) d t|f(y)| d \mu_{\lambda}(y)
\end{aligned}
$$

and the last double integral converges. Further, an application of (2.9) gives

$$
\begin{aligned}
\int_{0}^{1}\left|W_{t}^{\lambda} f(x)-\chi_{\{\lambda \leq 0\}} W_{t}^{\lambda} f(0)\right| \frac{d t}{\sqrt{t}} \\
\leq C_{\lambda, a, x} \int_{0 a}^{1} \int_{a}^{b}\left(\frac{(x y)^{-\lambda}}{\sqrt{t}} e^{-(x-y)^{2} / 4 t}+\frac{\chi_{\{\lambda \leq 0\}}}{t^{\lambda+1 / 2}} e^{-y^{2} / 4 t}\right)|f(y)| d \mu_{\lambda}(y) \frac{d t}{\sqrt{t}} \\
\leq C_{\lambda, a, b, x} \int_{a}^{b}\left\{\frac{1}{|x-y|^{1 / 2}} \int_{0}^{1}\left(\frac{(x-y)^{2}}{t}\right)^{1 / 4} e^{-(x-y)^{2} / 4 t} \frac{d t}{t^{3 / 4}}\right. \\
\left.\quad+\chi_{\{\lambda \leq 0\}} \frac{1}{y^{1 / 2}} \int_{0}^{1}\left(\frac{y^{2}}{t}\right)^{1 / 4} e^{-y^{2} / 4 t} \frac{d t}{t^{\lambda+3 / 4}}\right\}|f(y)| d y .
\end{aligned}
$$

The last expression is controlled, up to a multiplicative constant, by the convergent integral $\int_{a}^{b}\left(|x-y|^{-1 / 2}+\chi_{\{\lambda \leq 0\}} y^{-1 / 2}\right)|f(y)| d y$. Combining the above facts we conclude that the integral defining $\Delta_{\lambda}^{-1 / 2} f(x)$ converges absolutely. 
Proposition 4.2. Let $\lambda>-1 / 2$. For $f \in C_{c}^{\infty}\left(\mathbb{R}_{+}\right)$the function $\Delta_{\lambda}^{-1 / 2} f$ is differentiable and

$$
R_{\lambda} f(x):=D \Delta_{\lambda}^{-1 / 2} f(x)=\text { p.v. } \int_{0}^{\infty} R_{\lambda}(x, y) f(y) d \mu_{\lambda}(y), \quad x>0,
$$

with the kernel given by

$$
R_{\lambda}(x, y)=\frac{1}{\sqrt{\pi}} \int_{0}^{\infty} \frac{\partial}{\partial x} W_{t}^{\lambda}(x, y) \frac{d t}{\sqrt{t}}, \quad x, y>0, x \neq y .
$$

Proof. Our reasoning is based on a comparison with the classical setting, which allows us to control the essential singularity. Let $\mathcal{H}$ denote the Hilbert transform and let $f \in C_{c}^{\infty}(\mathbb{R})$. It is well known that

$$
\begin{aligned}
\mathcal{H} f(x) & =\frac{1}{\pi} \text { p.v. } \int_{\mathbb{R}} \frac{f(y)}{y-x} d y \\
& =\text { p.v. } \int_{\mathbb{R}}\left(\frac{1}{\sqrt{\pi}} \int_{0}^{\infty} \frac{\partial}{\partial x} \mathcal{W}_{t}(x, y) \frac{d t}{\sqrt{t}}\right) f(y) d y \\
& =\frac{1}{\sqrt{\pi}} \frac{\partial}{\partial x} \int_{0}^{\infty}\left(\mathcal{W}_{t} f(x)-\mathcal{W}_{t} f(0)\right) \frac{d t}{\sqrt{t}}, \quad x \in \mathbb{R} .
\end{aligned}
$$

Thus we decompose

$$
\begin{aligned}
D \Delta_{\lambda}^{-1 / 2} f(x)= & \frac{1}{\sqrt{\pi}} x^{-\lambda} \frac{\partial}{\partial x} \int_{0}^{\infty}\left(\mathcal{W}_{t}\left(y^{\lambda} f\right)(x)-\mathcal{W}_{t}\left(y^{\lambda} f\right)(0)\right) \frac{d t}{\sqrt{t}} \\
& -\frac{\lambda}{\sqrt{\pi}} x^{-\lambda-1} \int_{0}^{\infty}\left(\mathcal{W}_{t}\left(y^{\lambda} f\right)(x)-\mathcal{W}_{t}\left(y^{\lambda} f\right)(0)\right) \frac{d t}{\sqrt{t}} \\
& +\frac{1}{\sqrt{\pi}} \frac{\partial}{\partial x} \int_{0}^{\infty}\left(W_{t}^{\lambda} f(x)-\chi_{\{\lambda \leq 0\}} W_{t}^{\lambda} f(0)\right. \\
& \left.-x^{-\lambda} \mathcal{W}_{t}\left(y^{\lambda} f\right)(x)+x^{-\lambda} \mathcal{W}_{t}\left(y^{\lambda} f\right)(0)\right) \frac{d t}{\sqrt{t}} \\
\equiv & \mathcal{I}_{1}-\mathcal{I}_{2}+\mathcal{I}_{3} .
\end{aligned}
$$

In view of 4.1) it follows that

$$
\mathcal{I}_{1}=\text { p.v. } \int_{0}^{\infty}\left(\frac{1}{\sqrt{\pi}} \int_{0}^{\infty} \frac{\partial}{\partial x} \mathcal{W}_{t}(x, y) \frac{d t}{\sqrt{t}}\right)(x y)^{-\lambda} f(y) d \mu_{\lambda}(y) ;
$$

this term contains the crucial singularity and, as we shall see, no singular integrals emerge from $\mathcal{I}_{2}$ and $\mathcal{I}_{3}$.

Next, we prove that it is legitimate to pass with $\partial / \partial x$ under the integral sign in $\mathcal{I}_{3}$. This task, however, is directly reduced to showing that, for any 
fixed $0<a<b<\infty$, the quantities

$$
\begin{aligned}
& \mathcal{J}_{1}(t)=\sup _{x \in[a, b]}\left|\mathcal{W}_{t}\left(y^{\lambda} f\right)(x)-\mathcal{W}_{t}\left(y^{\lambda} f\right)(0)\right|, \\
& \mathcal{J}_{2}(t)=\sup _{x \in[a, b]}\left|\frac{\partial}{\partial x} W_{t}^{\lambda} f(x)-x^{-\lambda} \frac{\partial}{\partial x} \mathcal{W}_{t}\left(y^{\lambda} f\right)(x)\right|,
\end{aligned}
$$

can be majorized by functions of $t$ belonging to $L^{1}\left(\mathbb{R}_{+}, t^{-1 / 2} d t\right)$. In addition, without any loss of generality, it may be assumed that supp $f \subset[a, b]$, with $0<a<b<\infty$, and $\left\|y^{\lambda} f\right\|_{\infty} \leq 1,\|f\|_{\infty} \leq 1$.

Observe that

$$
\mathcal{J}_{1}(t) \leq \frac{1}{t^{1 / 2}} \sup _{x \in[a, b]} \int_{a}^{b}\left|e^{-(x-y)^{2} / 4 t}-e^{-y^{2} / 4 t}\right| d y .
$$

For $t \geq 1$ and $x, y \in[a, b]$ we have

$$
\left|e^{-(x-y)^{2} / 4 t}-e^{-y^{2} / 4 t}\right|=\left|\frac{y^{2}}{4 t}-\frac{(x-y)^{2}}{4 t}+\mathcal{O}\left(\frac{(x-y)^{4}}{t^{2}}\right)-\mathcal{O}\left(\frac{y^{4}}{t^{2}}\right)\right| \leq \frac{C_{b}}{t} .
$$

Also, since for all $t>0$ and $x, y \in[a, b]$,

$$
\left|e^{-(x-y)^{2} / 4 t}-e^{-y^{2} / 4 t}\right| \leq e^{-(x-y)^{2} / 4 t}+e^{-a^{2} / 4 t},
$$

we get

$$
\frac{1}{t^{1 / 2}} \int_{a}^{b}\left|e^{-(x-y)^{2} / 4 t}-e^{-y^{2} / 4 t}\right| d y \leq \frac{2}{t^{1 / 2}} \int_{-\infty}^{\infty} e^{-u^{2} / 4 t} d u=4 \sqrt{\pi}
$$

Then

$$
\mathcal{J}_{1}(t) \leq C_{b} \min \left(t^{-3 / 2}, 1\right) \in L^{1}\left(\mathbb{R}_{+}, t^{-1 / 2} d t\right) .
$$

The treatment of $\mathcal{J}_{2}$ is not as straightforward. We consider two cases.

CASE 1: $t \leq b^{2}$. By 2.7 we get

$$
\begin{aligned}
\frac{\partial}{\partial x} W_{t}^{\lambda}(x, y) & \\
= & \frac{1}{(2 t)^{\lambda}} e^{-x y / 2 t} \frac{\partial}{\partial x}\left(\frac{1}{\sqrt{2 t}} e^{-(x-y)^{2} / 4 t}\right)\left(\frac{x y}{2 t}\right)^{-\lambda+1 / 2} I_{\lambda-1 / 2}\left(\frac{x y}{2 t}\right) \\
& \quad-\frac{y}{(2 t)^{\lambda+3 / 2}}\left(\frac{x y}{2 t}\right)^{-\lambda+1 / 2}\left(I_{\lambda-1 / 2}\left(\frac{x y}{2 t}\right)-I_{\lambda+1 / 2}\left(\frac{x y}{2 t}\right)\right) e^{-\left(x^{2}+y^{2}\right) / 4 t} \\
\equiv & H_{\lambda, 1}(t, x, y)-H_{\lambda, 2}(t, x, y), \quad t, x, y>0 .
\end{aligned}
$$

Next observe that (2.9) implies 


$$
\begin{aligned}
& H_{\lambda, 1}(t, x, y) \\
& \quad=\frac{1}{\sqrt{2 \pi}} \frac{\partial}{\partial x}\left(\frac{1}{\sqrt{2 t}} e^{-(x-y)^{2} / 4 t}\right)(x y)^{-\lambda}\left(1+\mathcal{O}\left(\frac{t}{x y}\right)\right) \\
& \quad=(x y)^{-\lambda} \frac{\partial}{\partial x} \mathcal{W}_{t}(x, y)-\frac{(x y)^{-\lambda}}{t^{3 / 2}}(x-y) e^{-(x-y)^{2} / 4 t} \mathcal{O}\left(\frac{t}{x y}\right), \quad x, y \in[a, b] .
\end{aligned}
$$

In order to analyze $H_{\lambda, 2}(t, x, y)$ we use again $(2.9)$ and obtain

$$
\left|H_{\lambda, 2}(t, x, y)\right|=\frac{y}{t^{\lambda+3 / 2}} e^{-(x-y)^{2} / 4 t}\left(\frac{x y}{2 t}\right)^{-\lambda} \mathcal{O}\left(\frac{t}{x y}\right), \quad x, y \in[a, b] .
$$

Thus $H_{\lambda, 1}$ and $H_{\lambda, 2}$ satisfy

$$
\begin{aligned}
\left|H_{\lambda, 1}(t, x, y)-(x y)^{-\lambda} \frac{\partial}{\partial x} \mathcal{W}_{t}(x, y)\right| & \leq C_{\lambda, a, b} \frac{1}{t^{1 / 2}} e^{-(x-y)^{2} / 4 t}, \quad x, y \in[a, b], \\
\left|H_{\lambda, 2}(t, x, y)\right| & \leq C_{\lambda, a, b} \frac{1}{t^{1 / 2}} e^{-(x-y)^{2} / 4 t}, \quad x, y \in[a, b] .
\end{aligned}
$$

Consequently,

$$
\begin{gathered}
\int_{a}^{b}\left|H_{\lambda, 1}(t, x, y)-(x y)^{-\lambda} \frac{\partial}{\partial x} \mathcal{W}_{t}(x, y)\right||f(y)| d \mu_{\lambda}(y) \\
\leq C_{\lambda, a, b} \frac{1}{t^{1 / 2}} \int_{-\infty}^{\infty} e^{-(x-y)^{2} / 4 t} d y=C_{\lambda, a, b}
\end{gathered}
$$

and

$$
\int_{a}^{b}\left|H_{\lambda, 2}(t, x, y)\right||f(y)| d \mu_{\lambda}(y)=C_{\lambda, a, b}
$$

Hence $\mathcal{J}_{2}(t) \leq C_{\lambda, a, b}$ when $t \leq b^{2}$.

CASe 2: $t>a^{2}$. We deduce from (2.7) that

$$
\begin{aligned}
\frac{\partial}{\partial x} W_{t}^{\lambda}(x, y)= & \frac{1}{(2 t)^{\lambda+1 / 2}} e^{-\left(x^{2}+y^{2}\right) / 4 t}\left(x\left(\frac{y}{2 t}\right)^{2}\left(\frac{x y}{2 t}\right)^{-\lambda-1 / 2} I_{\lambda+1 / 2}\left(\frac{x y}{2 t}\right)\right. \\
& \left.-\frac{x}{2 t}\left(\frac{x y}{2 t}\right)^{-\lambda+1 / 2} I_{\lambda-1 / 2}\left(\frac{x y}{2 t}\right)\right) .
\end{aligned}
$$

Then 2.8 implies

$$
\left|\frac{\partial}{\partial x} W_{t}^{\lambda}(x, y)\right| \leq C_{\lambda, a, b} \frac{x}{t^{\lambda+3 / 2}} e^{-\left(x^{2}+y^{2}\right) / 4 t}\left(\frac{y^{2}}{t}+1\right) \leq \frac{C_{\lambda, a, b}}{t^{\lambda+3 / 2}}, \quad x, y \in[a, b] .
$$

Therefore

$$
\int_{a}^{b}\left|\frac{\partial}{\partial x} W_{t}^{\lambda}(x, y) f(y)\right| d \mu_{\lambda}(y) \leq \frac{C_{\lambda, a, b}}{t^{\lambda+3 / 2}}, \quad x \in[a, b] .
$$


On the other hand, for every $x \in[a, b]$,

$$
\begin{aligned}
\int_{a}^{b}(x y)^{-\lambda}\left|\frac{\partial}{\partial x} \mathcal{W}_{t}(x, y) f(y)\right| d \mu_{\lambda}(y) & \\
& \leq \int_{a}^{b}(x y)^{-\lambda} \frac{|x-y|}{t^{3 / 2}} e^{-(x-y)^{2} / 4 t}|f(y)| d \mu_{\lambda}(y) \leq \frac{C_{\lambda, a, b}}{t^{3 / 2}} .
\end{aligned}
$$

In this way we conclude that $\mathcal{J}_{2}(t) \leq C_{\lambda, a, b} t^{-1}$ for $t>a^{2}$.

A combination of Cases 1 and 2 reveals that

$$
\mathcal{J}_{2}(t) \leq C_{\lambda, a, b} \min \left(1, t^{-1}\right) \in L^{1}\left(\mathbb{R}_{+}, t^{-1 / 2} d t\right) .
$$

Now passing with $\partial / \partial x$ under the integral in $\mathcal{I}_{3}$ is justified and we get

$$
\begin{aligned}
D \Delta_{\lambda}^{-1 / 2} f(x)= & \text { p.v. } \int_{0}^{\infty}\left(\frac{1}{\sqrt{\pi}} \int_{0}^{\infty} \frac{\partial}{\partial x} \mathcal{W}_{t}(x, y) \frac{d t}{\sqrt{t}}\right)(x y)^{-\lambda} f(y) d \mu_{\lambda}(y) \\
& +\frac{1}{\sqrt{\pi}} \int_{0}^{\infty}\left(\frac{\partial}{\partial x} W_{t}^{\lambda} f(x)-x^{-\lambda} \frac{\partial}{\partial x} \mathcal{W}_{t}\left(y^{\lambda} f\right)(x)\right) \frac{d t}{\sqrt{t}} \\
\equiv & \mathcal{I}_{4}+\mathcal{I}_{5} .
\end{aligned}
$$

Having in mind the estimates obtained so far in this proof, it is straightforward to check that

$$
\mathcal{I}_{5}=\frac{1}{\sqrt{\pi}} \int_{0}^{\infty} \int_{0}^{\infty}\left(\frac{\partial}{\partial x} W_{t}^{\lambda}(x, y)-(x y)^{-\lambda} \frac{\partial}{\partial x} \mathcal{W}_{t}(x, y)\right) f(y) d \mu_{\lambda}(y) \frac{d t}{\sqrt{t}}
$$

and that the double integral above converges absolutely for any fixed $x>0$. Thus the order of integration may be switched and then the cancellations occurring between $\mathcal{I}_{4}$ and $\mathcal{I}_{5}$ lead to the desired principal value integral representation of $D \Delta_{\lambda}^{-1 / 2} f(x)$. Notice that the differentiability of $\Delta_{\lambda}^{-1 / 2} f$ is implicitly contained in the whole reasoning.

It is perhaps worth mentioning that the kernel $R_{\lambda}(x, y)$ can be expressed explicitly in terms of the Gauss hypergeometric function ${ }_{2} F_{1}$ (for the definition see for instance [12, Chapter 9]). More precisely, by means of (2.7) and the integral formula (cf. [15, 2.15.3 (2)])

$$
\begin{aligned}
& \int_{0}^{\infty} z^{1 / 2} e^{-\alpha z} I_{\nu}(\beta z) d z \\
& \quad=\alpha^{-\nu-3 / 2}\left(\frac{\beta}{2}\right)^{\nu} \frac{\Gamma(\nu+3 / 2)}{\Gamma(\nu+1)}{ }_{2} F_{1}\left(\frac{\nu+3 / 2}{2}, \frac{\nu+5 / 2}{2} ; \nu+1 ; \frac{\beta^{2}}{\alpha^{2}}\right),
\end{aligned}
$$

valid for $\nu>-3 / 2$ and $\alpha>\beta>0$, one computes that for $x \neq y$, 


$$
\begin{aligned}
R_{\lambda}(x, y) & \\
= & \frac{2}{\sqrt{\pi}} \frac{\Gamma(\lambda+2)}{\Gamma(\lambda+3 / 2)}(x y)^{-\lambda-1}\left\{y \Phi_{x, y}^{\lambda+2}{ }_{2} F_{1}\left(\frac{\lambda+2}{2}, \frac{\lambda+3}{2} ; \frac{2 \lambda+3}{2} ; 4 \Phi_{x, y}^{2}\right)\right. \\
& \left.-\frac{\lambda+1 / 2}{\lambda+1} x \Phi_{x, y}^{\lambda+1}{ }_{2} F_{1}\left(\frac{\lambda+1}{2}, \frac{\lambda+2}{2} ; \frac{2 \lambda+1}{2} ; 4 \Phi_{x, y}^{2}\right)\right\},
\end{aligned}
$$

with $\Phi_{x, y}=x y /\left(x^{2}+y^{2}\right)$. This representation, even though explicit, does not seem to be convenient for performing necessary kernel estimates since there are essential cancellations between the two terms containing ${ }_{2} F_{1}$ functions with different parameters. For $\lambda=0$ the above expression can be simplified (the relevant property of ${ }_{2} F_{1}$ can be found in [12, Section 9.8]) and we have

$$
R_{0}(x, y)=\frac{1}{\pi}\left(\frac{1}{y-x}-\frac{1}{y+x}\right), \quad x \neq y .
$$

Note that the same result can be obtained more directly since $W_{t}^{0}(x, y)=$ $\mathcal{W}_{t}(x, y)+\mathcal{W}_{t}(x,-y)$ (this identity follows from the fact that $I_{-1 / 2}(z)=$ $\sqrt{2 / \pi z} \cosh z$, cf. [12, (5.8.5)]) and the conclusion is obtained by a comparison with the Hilbert transform kernel. Finally, by 4.3 we see that $R_{0} f$ coincides with the Hilbert transform of the even extension of $f$, restricted to the positive half-line.

The following estimates for the kernel $R_{\lambda}(x, y)$ will be crucial in proving Theorem 2.3. They can also be obtained as consequences of [1, (1.6)], but our procedure, via the heat kernel, is different from that contained in [10, Lemma 2.1], [11, Theorem 2.1] and proving the estimate in [1].

Lemma 4.3. Let $\lambda>-1 / 2$. Then for all $x, y>0, x \neq y$, the integral defining $R_{\lambda}(x, y)$ converges absolutely and we have

$$
R_{\lambda}(x, y)=\frac{1}{\pi} \frac{(x y)^{-\lambda}}{y-x}+\mathcal{O}\left(y^{-2 \lambda-1}\left(1+\log \frac{x y}{(y-x)^{2}}\right)\right), \quad x / 2<y<2 x .
$$

Moreover, in the off-diagonal region,

$$
\left|R_{\lambda}(x, y)\right| \leq C_{\lambda} \begin{cases}x^{-2 \lambda-1}, & y \leq x / 2 \\ x y^{-2 \lambda-2}, & 2 x \leq y\end{cases}
$$

Proof. Observe first that by (2.7),

$$
\begin{aligned}
\frac{\partial}{\partial x} W_{t}^{\lambda}(x, y) & \\
= & \frac{1}{(2 t)^{\lambda+1 / 2}}\left(x\left(\frac{y}{2 t}\right)^{2}\left(\frac{x y}{2 t}\right)^{-\lambda-1 / 2} I_{\lambda+1 / 2}\left(\frac{x y}{2 t}\right)\right. \\
& \left.-\frac{x}{2 t}\left(\frac{x y}{2 t}\right)^{-\lambda+1 / 2} I_{\lambda-1 / 2}\left(\frac{x y}{2 t}\right)\right) e^{-\left(x^{2}+y^{2}\right) / 4 t}, \quad t, x, y>0 .
\end{aligned}
$$


Now assume that $0<x / 2<y<2 x$ and $x \neq y$. We write

$$
\begin{aligned}
\sqrt{\pi} R_{\lambda}(x, y) & =\int_{0}^{x y} \frac{\partial}{\partial x} W_{t}^{\lambda}(x, y) \frac{d t}{\sqrt{t}}+\int_{x y}^{\infty} \frac{\partial}{\partial x} W_{t}^{\lambda}(x, y) \frac{d t}{\sqrt{t}} \\
& \equiv R_{\lambda}^{1}(x, y)+R_{\lambda}^{2}(x, y) .
\end{aligned}
$$

By (4.4) and 2.8) it follows that

$$
\begin{aligned}
& \left|R_{\lambda}^{2}(x, y)\right| \\
& \quad \leq C_{\lambda} \int_{x y}^{\infty}\left(\frac{x y^{2}}{t^{2}}+\frac{x}{t}\right) \frac{d t}{t^{\lambda+1}} \leq C_{\lambda}\left(\frac{x y^{2}}{(x y)^{\lambda+2}}+\frac{x}{(x y)^{\lambda+1}}\right) \leq C_{\lambda} y^{-2 \lambda-1} .
\end{aligned}
$$

Next, by (4.4) and $(2.9)$ we get

$$
\begin{aligned}
R_{\lambda}^{1}(x, y)= & \int_{0}^{x y} \frac{2^{-\lambda-1 / 2}}{t^{\lambda+1}}\left(\frac{x y}{2 t}\right)^{-\lambda}\left(\frac{y}{2 t}\left(\frac{x y}{2 t}\right)^{1 / 2} I_{\lambda+1 / 2}\left(\frac{x y}{2 t}\right)\right. \\
& \left.-\frac{x}{2 t}\left(\frac{x y}{2 t}\right)^{1 / 2} I_{\lambda-1 / 2}\left(\frac{x y}{2 t}\right)\right) e^{-\left(x^{2}+y^{2}\right) / 4 t} d t \\
= & \frac{1}{2 \sqrt{2}} \int_{0}^{x y} \frac{(x y)^{-\lambda}}{t^{2}}\left(\frac{y-x}{\sqrt{2 \pi}}+\mathcal{O}\left(\frac{t}{y}\right)\right) e^{-(x-y)^{2} / 4 t} d t \\
= & \frac{1}{4 \sqrt{\pi}}(x y)^{-\lambda}(y-x) \int_{0}^{\infty} \frac{1}{t^{2}} e^{-(x-y)^{2} / 4 t} d t \\
& -\frac{1}{4 \sqrt{\pi}}(x y)^{-\lambda}(y-x) \int_{x y}^{\infty} \frac{1}{t^{2}} e^{-(x-y)^{2} / 4 t} d t \\
& +(x y)^{-\lambda} \int_{0}^{x y} \frac{1}{t^{2}} \mathcal{O}\left(\frac{t}{y}\right) e^{-(x-y)^{2} / 4 t} d t \\
\equiv & K_{1}^{\lambda}(x, y)+K_{2}^{\lambda}(x, y)+K_{3}^{\lambda}(x, y) .
\end{aligned}
$$

We analyze each $K_{i}^{\lambda}(x, y), i=1,2,3$, separately. By a direct computation

$$
K_{1}^{\lambda}(x, y)=\frac{1}{\sqrt{\pi}} \frac{(x y)^{-\lambda}}{y-x} .
$$

To estimate $K_{2}^{\lambda}(x, y)$ we write

$$
\begin{aligned}
& \left|K_{2}^{\lambda}(x, y)\right| \\
& \quad \leq(x y)^{-\lambda}|y-x| \int_{x y}^{\infty} e^{-(x-y)^{2} / 4 t} \frac{d t}{t^{2}} \leq(x y)^{-\lambda} x \int_{x y}^{\infty} \frac{d t}{t^{2}} \leq C_{\lambda} y^{-2 \lambda-1} .
\end{aligned}
$$


In the case of $K_{3}^{\lambda}(x, y)$ one has

$$
\begin{aligned}
& \left|K_{3}^{\lambda}(x, y)\right| \\
& \quad \leq C_{\lambda}(x y)^{-\lambda} \frac{1}{y} \int_{0}^{x y} \frac{1}{t} e^{-(x-y)^{2} / 4 t} d t \leq C_{\lambda} y^{-2 \lambda-1} \int_{(x-y)^{2} / 4 x y}^{\infty} \frac{1}{u} e^{-u} d u,
\end{aligned}
$$

and splitting the last integral according to $u<1$ and $u \geq 1$ makes it clear that

$$
\left|K_{3}^{\lambda}(x, y)\right| \leq C_{\lambda} y^{-2 \lambda-1}\left(1+\log \frac{x y}{(x-y)^{2}}\right) .
$$

This completes the proof of the diagonal estimate of the lemma.

In order to justify the remaining estimates it suffices to bound suitably $R_{\lambda}^{i}(x, y), i=1,2$, in the off-diagonal region. Notice that 2.8$)$, together with (4.4), implies

$$
\begin{aligned}
\left|R_{\lambda}^{2}(x, y)\right| \leq & C_{\lambda} x \int_{x y}^{\infty} \frac{1}{t^{\lambda+2}}\left(\frac{y^{2}}{t}+1\right) e^{-\left(x^{2}+y^{2}\right) / 4 t} d t \\
\leq & C_{\lambda} x\left(\frac{y^{2}}{\left(x^{2}+y^{2}\right)^{\lambda+2}} \int_{0}^{\left(x^{2}+y^{2}\right) / 4 x y} u^{\lambda+1} e^{-u} d u\right. \\
& \left.+\frac{1}{\left(x^{2}+y^{2}\right)^{\lambda+1}} \int_{0}^{\left(x^{2}+y^{2}\right) / 4 x y} u^{\lambda} e^{-u} d u\right) \\
\leq & C_{\lambda} \frac{x}{\left(x^{2}+y^{2}\right)^{\lambda+1}} .
\end{aligned}
$$

From this we easily obtain the desired estimates, with $R_{\lambda}(x, y)$ replaced by $R_{\lambda}^{2}(x, y)$. Finally, by applying 2.9 together with (4.4),

$$
\begin{aligned}
\left|R_{\lambda}^{1}(x, y)\right| & \leq C_{\lambda}(x y)^{-\lambda}(x+y) \int_{0}^{x y} e^{-(x-y)^{2} / 4 t} \frac{d t}{t^{2}} \\
& \leq C_{\lambda} \frac{(x y)^{-\lambda}}{|x-y|^{2 \lambda+3}} \int_{0}^{x y} t^{\lambda} d t \leq C_{\lambda} \frac{x y}{|x-y|^{2 \lambda+3}}
\end{aligned}
$$

and the off-diagonal estimates follow again, this time for $R_{\lambda}^{1}(x, y)$. The proof of Lemma 4.3 is finished.

We now show that the off-diagonal estimates of Lemma 4.3 are sharp in certain regions.

Lemma 4.4. Let $\lambda>-1 / 2$. There exist $b>1$ and $a$ (positive) constant $c_{\lambda}$ such that 


$$
\begin{array}{ll}
R_{\lambda}(x, y) \leq-c_{\lambda} x^{-2 \lambda-1}, & 0<y \leq x / b, \\
R_{\lambda}(x, y) \geq c_{\lambda} x y^{-2 \lambda-2}, & 0<b x \leq y .
\end{array}
$$

Proof. Using (4.4) and then performing the change of variable $u=2 t / x^{2}$ we obtain

$$
\begin{aligned}
R_{\lambda}(x, y)= & \frac{1}{2^{\lambda+1 / 2}} \int_{0}^{\infty}\left(x\left(\frac{y}{2 t}\right)^{2}\left(\frac{x y}{2 t}\right)^{-\lambda-1 / 2} I_{\lambda+1 / 2}\left(\frac{x y}{2 t}\right)\right. \\
& \left.-\frac{x}{2 t}\left(\frac{x y}{2 t}\right)^{-\lambda+1 / 2} I_{\lambda-1 / 2}\left(\frac{x y}{2 t}\right)\right) e^{-\left(x^{2}+y^{2}\right) / 4 t} \frac{d t}{t^{\lambda+1}} \\
= & \frac{1}{\sqrt{2} x^{2 \lambda+1}} \int_{0}^{\infty} \frac{1}{u^{\lambda+1}}\left(\left(\frac{z}{u}\right)^{2}\left(\frac{z}{u}\right)^{-\lambda-1 / 2} I_{\lambda+1 / 2}\left(\frac{z}{u}\right)\right. \\
& \left.-\frac{1}{u}\left(\frac{z}{u}\right)^{-\lambda+1 / 2} I_{\lambda-1 / 2}\left(\frac{z}{u}\right)\right) e^{-\left(1+z^{2}\right) / 2 u} d u, \quad x, y>0,
\end{aligned}
$$

where $z=y / x$. Then, with the aid of (2.8) and the dominated convergence theorem (a suitable integrable majorant can be derived by means of 2.8 and (2.9) $)$, we see that

$$
\lim _{z \rightarrow 0^{+}} x^{2 \lambda+1} R_{\lambda}(x, y)=-\frac{1}{2^{\lambda} \Gamma(\lambda+1 / 2)} \int_{0}^{\infty} \frac{1}{u^{\lambda+2}} e^{-1 / 2 u} d u=-\frac{2 \Gamma(\lambda+1)}{\Gamma(\lambda+1 / 2)},
$$

and the desired bound for $y<x / b$ follows. Similarly, changing the variable $u=2 t / y^{2}$, we can write

$$
\begin{aligned}
R_{\lambda}(x, y)= & \frac{x}{\sqrt{2} y^{2 \lambda+2}} \int_{0}^{\infty} \frac{1}{u^{\lambda+1}}\left(\frac{1}{u^{2}}\left(\frac{z}{u}\right)^{-\lambda-1 / 2} I_{\lambda+1 / 2}\left(\frac{z}{u}\right)\right. \\
& \left.-\frac{1}{u}\left(\frac{z}{u}\right)^{-\lambda+1 / 2} I_{\lambda-1 / 2}\left(\frac{z}{u}\right)\right) e^{-\left(1+z^{2}\right) / 2 u} d u, \quad x, y>0,
\end{aligned}
$$

where now $z=x / y$, thus, again in view of 2.8 and the dominated convergence theorem,

$$
\begin{aligned}
& \lim _{z \rightarrow 0^{+}} \frac{y^{2 \lambda+2}}{x} R_{\lambda}(x, y) \\
= & \frac{1}{2^{\lambda+1} \Gamma(\lambda+1 / 2)} \int_{0}^{\infty} \frac{1}{u^{\lambda+2}}\left(\frac{1}{(\lambda+1 / 2) u}-2\right) e^{-1 / 2 u} d u=\frac{\Gamma(\lambda+1)}{\Gamma(\lambda+3 / 2)} .
\end{aligned}
$$

This gives the remaining bound for $b x<y$.

Consider the auxiliary operator

$$
\mathcal{H}_{\lambda, \mathrm{loc}} f(x)=\frac{1}{\pi} \mathrm{p} . \mathrm{v} \cdot \int_{x / 2}^{2 x} \frac{(x y)^{-\lambda}}{y-x} f(y) d \mu_{\lambda}(y), \quad x>0,
$$


defined for, say, $f \in C_{c}^{\infty}\left(\mathbb{R}_{+}\right)$. Note that the principal value integral converges for a.e. $x>0$ if only $f$ is locally integrable; this follows by the relation with the Hilbert transform.

According to [2, Lemma 1], the local Hilbert transform $\mathcal{H}_{0, \text { loc }}$ is bounded on $L^{2}\left(\mathbb{R}_{+}, d x\right)$ (this fact can also be proved directly by combining the $L^{2}$ boundedness of the Hilbert transform with the classical Hardy inequalities). Consequently, applying Lemma 2.8 to the scalar-valued operator $T=\mathcal{H}_{0, \text { loc }}$ we get the following.

Lemma 4.5. Let $\lambda \in \mathbb{R}$. Then $\mathcal{H}_{\lambda, \text { loc }}$ is a local Calderón-Zygmund operator, hence, for each $\delta \in \mathbb{R}$, it is bounded on $L^{p}\left(\mathbb{R}_{+}, x^{\delta} d x\right), 1<p<\infty$, and from $L^{1}\left(\mathbb{R}_{+}, x^{\delta} d x\right)$ into $L^{1, \infty}\left(\mathbb{R}_{+}, x^{\delta} d x\right)$.

We are now prepared to give the main proof.

Proof of Theorem 2.3. We shall justify the sufficiency parts first. To this end assume that $f \in C_{c}^{\infty}\left(\mathbb{R}_{+}\right)$. Similarly to the proof of Theorem 2.1. we split the Riesz operator

$$
\begin{aligned}
R_{\lambda} f(x) & =\left\{\int_{0}^{x / 2}+\text { p.v. } \int_{x / 2}^{2 x}+\int_{2 x}^{\infty}\right\} R_{\lambda}(x, y) f(y) d \mu_{\lambda}(y) \\
& \equiv R_{\lambda, 1} f(x)+R_{\lambda, 2} f(x)+R_{\lambda, 3} f(x) .
\end{aligned}
$$

The operators $R_{\lambda, 1}, R_{\lambda, 2}$ and $R_{\lambda, 3}$ will be analyzed separately. Note that only the diagonal part $R_{\lambda, 2}$ is given by a singular integral.

Applying Lemma 4.3 we obtain, for all $x>0$,

$$
\begin{aligned}
& \left|R_{\lambda, 1} f(x)\right| \leq C_{\lambda} \frac{1}{x^{2 \lambda+1}} \int_{0}^{x / 2}|f(y)| y^{2 \lambda} d y \leq C_{\lambda} H_{0}^{2 \lambda}|f|(x), \\
& \left|R_{\lambda, 3} f(x)\right| \leq C_{\lambda} x \int_{2 x}^{\infty}|f(y)| \frac{d y}{y^{2}} \leq C_{\lambda} H_{\infty}^{1}|f|(x),
\end{aligned}
$$

and also

$$
\left|R_{\lambda, 2} f(x)-\mathcal{H}_{\lambda, \operatorname{loc}} f(x)\right| \leq C_{\lambda} \int_{x / 2}^{2 x} \frac{1}{y}\left(1+\log \frac{x y}{(x-y)^{2}}\right)|f(y)| d y .
$$

Note that the operator $N$ defined by

$$
N f(x)=\int_{x / 2}^{2 x} \frac{1}{y}\left(1+\log \frac{x y}{(x-y)^{2}}\right) f(y) d y, \quad x>0,
$$

and occurring above, is bounded on $L^{p}\left(\mathbb{R}_{+}, x^{\delta} d x\right)$ for each $\delta \in \mathbb{R}$ and each $1 \leq p<\infty$. Indeed, observe first that the integral defining $N \mathbf{1}(x)$ is finite and in fact does not depend on $x>0$. Then, using Jensen's inequality and 
changing the order of integration, we get

$$
\begin{aligned}
\int_{0}^{\infty}|N f(x)|^{p} x^{\delta} d x & \leq C_{p} \int_{0}^{\infty} x^{\delta} \int_{x / 2}^{2 x} \frac{1}{y}\left(1+\log \frac{x y}{(x-y)^{2}}\right)|f(y)|^{p} d y d x \\
& \leq C_{p} \int_{0}^{\infty}|f(y)|^{p} \int_{y / 2}^{2 y} \frac{1}{x}\left(1+\log \frac{x y}{(x-y)^{2}}\right) x^{\delta} d x d y \\
& \leq C_{p, \delta} \int_{0}^{\infty}|f(y)|^{p} y^{\delta} d y .
\end{aligned}
$$

Taking into account Lemmas 2.6 and 2.7, the above facts and Lemma 4.5 , we deduce the following mapping properties of $R_{\lambda}$ considered on the space $\left(\mathbb{R}_{+}, x^{\delta} d x\right)$. For $1<p<\infty, R_{\lambda}$ is of strong type $(p, p)$ if $-1-p<\delta<$ $(2 \lambda+1) p-1$. If $-2 \leq \delta \leq 2 \lambda$ then $R_{\lambda}$ is of weak type $(1,1)$. Finally, $R_{\lambda}$ is of restricted weak type $(p, p)$ when $1<p<\infty$ and $-p-1 \leq \delta \leq$ $(2 \lambda+1) p-1$. These properties combined justify the sufficiency parts of Theorem 2.3 .

The necessity parts will be justified once we show the following statements (we assume that $1 \leq p<\infty$ and the underlying space is $\left(\mathbb{R}_{+}, x^{\delta} d x\right)$ ).

(A) If $R_{\lambda}$ is of restricted weak type $(p, p)$ then $-p-1 \leq \delta \leq(2 \lambda+1) p-1$.

(B1) $R_{\lambda}$ is not of weak type $(p, p)$ when $p>1$ and $\delta=-p-1$.

(B2) $R_{\lambda}$ is not of weak type $(p, p)$ when $p>1$ and $\delta=(2 \lambda+1) p-1$.

(C) $R_{\lambda}$ is not of strong type $(1,1)$ for $-2 \leq \delta \leq 2 \lambda$.

Item (A) can be deduced immediately by a standard interpolation argument, once we prove the other items. For item (B1) observe that, in view of the above considerations and (4.5), $R_{\lambda}$ is of weak type $(p, p)$ for $\delta=-p-1$, $p>1$, if and only if $R_{\lambda, 3}$ has the same property. Moreover, by Lemma 4.4 , if $R_{\lambda, 3}$ has this property then the operator $H_{\infty}^{1}$ also has it. But it is known (see [2, Theorem 5]) that $H_{\infty}^{1}$ fails to be of weak type $(p, p)$ for $\delta=-p-1, p>1$. Therefore the same negative result holds for $R_{\lambda}$; this gives (B1). Treatment of (B2) is similar: $R_{\lambda}$ is of weak type $(p, p)$ for $\delta=(2 \lambda+1) p-1, p>1$, if and only if $R_{\lambda, 1}$ has this property. Then, using Lemma 4.4, we infer that the property for $R_{\lambda, 1}$ implies the same for $H_{0}^{2 \lambda}$. However, it is known (see [1. Theorem 1]) that $H_{0}^{2 \lambda}$ does not have the property in question, thus (B2) follows.

It remains to show $(\mathrm{C})$. For $0<\varepsilon<1 / 4$ consider the function $f_{\varepsilon}(x)=$ $x^{-\lambda} \chi_{(1,1+\varepsilon)}(x), x>0$. By the estimate in the diagonal region from Lemma 4.3 we have 


$$
\begin{aligned}
\int_{0}^{\infty}\left|R_{\lambda} f_{\varepsilon}(x)\right| x^{\delta} d x & \geq \int_{1+2 \varepsilon}^{2}\left|\int_{x / 2}^{2 x} R_{\lambda}(x, y) f_{\varepsilon}(y) d \mu_{\lambda}(y)\right| x^{\delta} d x \\
& \geq \frac{1}{\pi} \int_{1+2 \varepsilon}^{2} x^{\delta-\lambda}\left|\int_{1}^{1+\varepsilon} \frac{d y}{y-x}\right| d x-C_{\lambda} \int_{1+2 \varepsilon}^{2} N f_{\varepsilon}(x) x^{\delta} d x
\end{aligned}
$$

Then, if $R_{\lambda}$ were bounded on $L^{1}\left(\mathbb{R}_{+}, x^{\delta} d x\right)$ we would have

$$
\begin{aligned}
\int_{1+2 \varepsilon}^{2} x^{\delta-\lambda}\left|\int_{1}^{1+\varepsilon} \frac{1}{y-x} d y\right| d x & \leq C_{\lambda}\left(\left\|R_{\lambda} f_{\varepsilon}\right\|_{L^{1}\left(\mathbb{R}_{+}, x^{\delta} d x\right)}+\left\|N f_{\varepsilon}\right\|_{L^{1}\left(\mathbb{R}_{+}, x^{\delta} d x\right)}\right) \\
& \leq C_{\lambda, \delta}\left\|f_{\varepsilon}\right\|_{L^{1}\left(\mathbb{R}_{+}, x^{\delta} d x\right)}
\end{aligned}
$$

and hence it would follow that

$$
\begin{aligned}
\varepsilon & \geq c_{\lambda, \delta}\left\|f_{\varepsilon}\right\|_{L^{1}\left(\mathbb{R}_{+}, x^{\delta} d x\right)} \\
& \geq c_{\lambda, \delta} \int_{1+2 \varepsilon}^{2} \log \frac{x-1}{x-1-\varepsilon} d x=c_{\lambda, \delta}\left(\varepsilon \log \frac{1}{4 \varepsilon}+(1-\varepsilon) \log \frac{1}{1-\varepsilon}\right) .
\end{aligned}
$$

But the inequality between the outer expressions cannot hold with $c_{\delta, \lambda}$ independent of $\varepsilon \in(0,1 / 4)$, as can be seen immediately by letting $\varepsilon \rightarrow 0^{+}$. Thus $R_{\lambda}$ is not bounded on $L^{1}\left(\mathbb{R}_{+}, x^{\delta} d x\right)$ and $(\mathrm{C})$ is justified.

The proof of Theorem 2.3 is now complete.

REMARK 4.6. The boundedness properties of the operator $\widetilde{R}_{\lambda}$ related to $\widetilde{\Delta}_{\lambda}$ can be proved by a careful analysis of the proof of Theorem 2.3. Split $\widetilde{R}_{\lambda}$ into three parts,

$$
\begin{aligned}
\widetilde{R}_{\lambda} f(x) & =\left\{\int_{0}^{x / 2}+\text { p.v. } \int_{x / 2}^{2 x}+\int_{2 x}^{\infty}\right\} \widetilde{R}_{\lambda}(x, y) f(y) d y \\
& \equiv \widetilde{R}_{\lambda, 1} f(x)+\widetilde{R}_{\lambda, 2} f(x)+\widetilde{R}_{\lambda, 3} f(x) .
\end{aligned}
$$

Since $\widetilde{R}_{\lambda}(x, y)=(x y)^{\lambda} R_{\lambda}(x, y)$, with the aid of Lemmas 4.3 and 4.4 the operators $\widetilde{R}_{\lambda, 1}$ and $\widetilde{R}_{\lambda, 3}$ can be controlled above and below by $H_{0}^{\lambda}$ and $H_{\infty}^{\lambda+1}$, respectively. Moreover, $\widetilde{R}_{\lambda, 2}$ is a local Calderón-Zygmund operator with respect to Lebesgue measure; this follows from the corresponding property for $R_{\lambda, 2}$ (see Lemmas 4.3 and 4.5). Thus we obtain similar results for $\widetilde{R}_{\lambda}$ to those stated in Theorem 2.3, just replacing the role of the interval $(-p-1,(2 \lambda+1) p-1)$ by $(-(\lambda+1) p-1,(\lambda+1) p-1)$.

5. The heat integral square function. This section is devoted to the proof of Theorem 2.5. Recall that the square function we take into account 
is given by

$$
g_{\lambda}(f)(x)=\left(\int_{0}^{\infty} t\left|\frac{\partial}{\partial t} \int_{0}^{\infty} W_{t}^{\lambda}(x, y) f(y) d \mu_{\lambda}(y)\right|^{2} d t\right)^{1 / 2}, \quad x>0 .
$$

We will need several technical results, one of them being the following important estimate.

Lemma 5.1. Let $\lambda>-1 / 2$. There exists a constant $C_{\lambda}$ such that for all $x, y>0$,

$$
\begin{aligned}
\left(\int_{0}^{\infty} t \mid \frac{\partial}{\partial t} W_{t}^{\lambda}(x, y)-\chi_{\{0<x / 2<y<2 x\}}(x y)^{-\lambda} \frac{\partial}{\partial t}\right. & \left.\left.\mathcal{W}_{t}(x, y)\right|^{2} d t\right)^{1 / 2} \\
& \leq C_{\lambda}(\max \{x, y\})^{-2 \lambda-1}
\end{aligned}
$$

Proof. We first show that the estimate holds in the diagonal region $0<$ $x / 2 \leq y \leq 2 x$. We shall consider two cases determined by the asymptotics at $0^{+}$and $\infty$ of the Bessel function involved.

CASE 1: $x y \geq t$. Observe that

$$
\begin{aligned}
\frac{\partial}{\partial t} W_{t}^{\lambda}(x, y)= & \frac{\partial}{\partial t}\left(\frac{1}{\sqrt{2 t}} e^{-(x-y)^{2} / 4 t}\right)(x y)^{-\lambda} e^{-x y / 2 t}\left(\frac{x y}{2 t}\right)^{1 / 2} I_{\lambda-1 / 2}\left(\frac{x y}{2 t}\right) \\
& +(x y)^{-\lambda} \frac{\partial}{\partial t}\left(e^{-x y / 2 t}\left(\frac{x y}{2 t}\right)^{1 / 2} I_{\lambda-1 / 2}\left(\frac{x y}{2 t}\right)\right) \frac{1}{\sqrt{2 t}} e^{-(x-y)^{2} / 4 t} \\
\equiv & E_{\lambda, 1}(t, x, y)+E_{\lambda, 2}(t, x, y) .
\end{aligned}
$$

Then, according to $(2.9)$, we get

$$
\begin{aligned}
E_{\lambda, 1}(t, x, y)= & \frac{(x y)^{-\lambda}}{\sqrt{2 \pi}} \frac{\partial}{\partial t}\left(\frac{1}{\sqrt{2 t}} e^{-(x-y)^{2} / 4 t}\right)\left(1+\mathcal{O}\left(\frac{t}{x y}\right)\right) \\
= & (x y)^{-\lambda} \frac{\partial}{\partial t} \mathcal{W}_{t}(x, y) \\
& -(x y)^{-\lambda} \mathcal{O}\left(\frac{t}{x y}\right)\left(\frac{1}{(2 t)^{3 / 2}}-\frac{1}{(2 t)^{5 / 2}}(x-y)^{2}\right) e^{-(x-y)^{2} / 4 t} \\
\equiv & (x y)^{-\lambda} \frac{\partial}{\partial t} \mathcal{W}_{t}(x, y)+E_{\lambda, 1}^{1}(t, x, y)+E_{\lambda, 1}^{2}(t, x, y) .
\end{aligned}
$$

Integrating the last two terms in $t \leq x y$ we obtain

$$
\begin{aligned}
\left(\int_{0}^{x y} t\left|E_{\lambda, 1}^{1}(t, x, y)\right|^{2} d t\right)^{1 / 2} & \leq C_{\lambda}(x y)^{-\lambda-1}\left(\int_{0}^{x y} e^{-(x-y)^{2} / 2 t} d t\right)^{1 / 2} \\
& \leq C_{\lambda}(x y)^{-\lambda-1 / 2}
\end{aligned}
$$




$$
\begin{aligned}
& \left(\int_{0}^{x y} t\left|E_{\lambda, 1}^{2}(t, x, y)\right|^{2} d t\right)^{1 / 2} \\
& \quad \leq C_{\lambda}(x y)^{-\lambda-1}\left(\int_{0}^{x y}\left(\frac{(x-y)^{2}}{t}\right)^{2} e^{-(x-y)^{2} / 2 t} d t\right)^{1 / 2} \leq C_{\lambda}(x y)^{-\lambda-1 / 2} .
\end{aligned}
$$

Next, we analyze $E_{\lambda, 2}(t, x, y)$. By using 2.7$)$ it follows that

$$
\begin{aligned}
& E_{\lambda, 2}(t, x, y) \\
&=(x y)^{-\lambda} \frac{\partial}{\partial t}\left(e^{-x y / 2 t}\left(\frac{x y}{2 t}\right)^{1 / 2} I_{\lambda-1 / 2}\left(\frac{x y}{2 t}\right)\right) \frac{1}{\sqrt{2 t}} e^{-(x-y)^{2} / 4 t} \\
&=\frac{(x y)^{-\lambda+1}}{2 \sqrt{2} t^{5 / 2}} e^{-(x-y)^{2} / 4 t}\left(e^{-x y / 2 t}\left(\frac{x y}{2 t}\right)^{1 / 2} I_{\lambda-1 / 2}\left(\frac{x y}{2 t}\right)\right. \\
&\left.-e^{-x y / 2 t} \frac{2 \lambda t}{x y}\left(\frac{x y}{2 t}\right)^{1 / 2} I_{\lambda-1 / 2}\left(\frac{x y}{2 t}\right)-e^{-x y / 2 t}\left(\frac{x y}{2 t}\right)^{1 / 2} I_{\lambda+1 / 2}\left(\frac{x y}{2 t}\right)\right) .
\end{aligned}
$$

Then applying 2.9 we see that

$$
\begin{aligned}
& E_{\lambda, 2}(t, x, y) \\
& =\frac{(x y)^{-\lambda+1}}{4 \sqrt{\pi} t^{5 / 2}} e^{-(x-y)^{2} / 4 t}\left\{\left(1-c(\lambda) \frac{t}{x y}+\mathcal{O}\left(\frac{t^{2}}{(x y)^{2}}\right)\right)\right. \\
& \left.\quad-\frac{2 \lambda t}{x y}\left(1-c(\lambda) \frac{t}{x y}+\mathcal{O}\left(\frac{t^{2}}{(x y)^{2}}\right)\right)-\left(1-c(\lambda+1) \frac{t}{x y}+\mathcal{O}\left(\frac{t^{2}}{(x y)^{2}}\right)\right)\right\},
\end{aligned}
$$

where $c(\nu)=[\nu-1 / 2,1]=(\nu-1 / 2)^{2}-1 / 4$. Hence, due to the occurring cancellations,

$$
\left|E_{\lambda, 2}(t, x, y)\right|=\frac{(x y)^{-\lambda+1}}{t^{5 / 2}} e^{-(x-y)^{2} / 4 t} \mathcal{O}\left(\frac{t^{2}}{(x y)^{2}}\right) .
$$

Consequently,

$$
\begin{aligned}
\left(\int_{0}^{x y} t\left|E_{\lambda, 2}(t, x, y)\right|^{2} d t\right)^{1 / 2} & \leq C_{\lambda}(x y)^{-\lambda-1}\left(\int_{0}^{x y} e^{-(x-y)^{2} / 2 t} d t\right)^{1 / 2} \\
& \leq C_{\lambda}(x y)^{-\lambda-1 / 2}
\end{aligned}
$$

In view of this and the previous estimates we conclude that for $x, y>0$,

$$
\left(\int_{0}^{x y} t\left|\frac{\partial}{\partial t} W_{t}^{\lambda}(x, y)-(x y)^{-\lambda} \frac{\partial}{\partial t} \mathcal{W}_{t}(x, y)\right|^{2} d t\right)^{1 / 2} \leq C_{\lambda}(x y)^{-\lambda-1 / 2}
$$

which finishes Case 1. 
CASE 2: $x y<t$. Observe that 2.7) leads to

$$
\begin{aligned}
\frac{\partial}{\partial t} W_{t}^{\lambda}(x, y) & \\
= & 2^{-\lambda-1 / 2} e^{-\left(x^{2}+y^{2}\right) / 4 t}\left(-\frac{\lambda+1 / 2}{t^{\lambda+3 / 2}}\left(\frac{x y}{2 t}\right)^{-\lambda+1 / 2} I_{\lambda-1 / 2}\left(\frac{x y}{2 t}\right)\right. \\
& -\frac{x y}{2 t^{\lambda+5 / 2}}\left(\frac{x y}{2 t}\right)^{-\lambda+1 / 2} I_{\lambda+1 / 2}\left(\frac{x y}{2 t}\right) \\
& \left.+\frac{x^{2}+y^{2}}{4 t^{\lambda+5 / 2}}\left(\frac{x y}{2 t}\right)^{-\lambda+1 / 2} I_{\lambda-1 / 2}\left(\frac{x y}{2 t}\right)\right) .
\end{aligned}
$$

Then 2.8) implies

$$
\begin{aligned}
\left|\frac{\partial}{\partial t} W_{t}^{\lambda}(x, y)\right| & \leq \frac{C_{\lambda}}{t^{\lambda+3 / 2}}\left(1+\frac{(x y)^{2}}{t^{2}}+\frac{x^{2}+y^{2}}{t}\right) e^{-\left(x^{2}+y^{2}\right) / 4 t} \\
& \leq \frac{C_{\lambda}}{t^{\lambda+3 / 2}} e^{-\left(x^{2}+y^{2}\right) / 8 t}
\end{aligned}
$$

hence we can write

$$
\begin{aligned}
\left(\int_{x y}^{\infty} t\left|\frac{\partial}{\partial t} W_{t}^{\lambda}(x, y)\right|^{2} d t\right)^{1 / 2} & \leq C_{\lambda}\left(\int_{x y}^{\infty} \frac{1}{t^{2 \lambda+2}} e^{-\left(x^{2}+y^{2}\right) / 4 t} d t\right)^{1 / 2} \\
& \leq \frac{C_{\lambda}}{\left(x^{2}+y^{2}\right)^{\lambda+1 / 2}}\left(\int_{0}^{\left(x^{2}+y^{2}\right) / 4 x y} u^{2 \lambda} e^{-u} d u\right)^{1 / 2} \\
& \leq C_{\lambda}(x y)^{-\lambda-1 / 2} .
\end{aligned}
$$

Moreover, by a straightforward computation,

$$
\left|\frac{\partial}{\partial t} \mathcal{W}_{t}(x, y)\right| \leq \frac{C}{t^{3 / 2}} e^{-(x-y)^{2} / 4 t}\left(1+\frac{(x-y)^{2}}{t}\right) \leq \frac{C}{t^{3 / 2}} e^{-(x-y)^{2} / 8 t}
$$

and therefore

$$
\begin{aligned}
\left(\int_{x y}^{\infty} t\left|(x y)^{-\lambda} \frac{\partial}{\partial t} \mathcal{W}_{t}(x, y)\right|^{2} d t\right)^{1 / 2} & \leq C(x y)^{-\lambda}\left(\int_{x y}^{\infty} \frac{1}{t^{2}} e^{-(x-y)^{2} / 4 t} d t\right)^{1 / 2} \\
& \leq C(x y)^{-\lambda-1 / 2}
\end{aligned}
$$

Now, combining the estimates of Cases 1 and 2, we conclude that for $0<x / 2 \leq y \leq 2 x$

$$
\begin{aligned}
\left(\int_{0}^{\infty} t\left|\frac{\partial}{\partial t} W_{t}^{\lambda}(x, y)-(x y)^{-\lambda} \frac{\partial}{\partial t} \mathcal{W}_{t}(x, y)\right|^{2} d t\right)^{1 / 2} & \leq C_{\lambda}(x y)^{-\lambda-1 / 2} \\
& \leq C_{\lambda}(\max \{x, y\})^{-2 \lambda-1}
\end{aligned}
$$


It remains to prove the relevant bound in the off-diagonal region. By symmetry of the kernel $W_{t}^{\lambda}(x, y)$ it is enough to focus on the cone $0<y<$ $x / 2$. As above, we consider two cases.

CASE 1: $x y \geq t$. According to (5.1) and (2.9),

$$
\begin{aligned}
\frac{\partial}{\partial t} W_{t}^{\lambda}(x, y) & \\
= & \frac{2^{-\lambda-1 / 2}}{\sqrt{2 \pi}} e^{-(x-y)^{2} / 4 t}\left\{-\frac{\lambda+1 / 2}{2^{-\lambda} t^{3 / 2}}(x y)^{-\lambda}\left(1+\mathcal{O}\left(\frac{t}{x y}\right)\right)\right. \\
& \left.-\frac{(x y)^{-\lambda+1}}{2^{-\lambda+1} t^{5 / 2}}\left(1+\mathcal{O}\left(\frac{t}{x y}\right)\right)+\frac{(x y)^{-\lambda}\left(x^{2}+y^{2}\right)}{2^{-\lambda+2} t^{5 / 2}}\left(1+\mathcal{O}\left(\frac{t}{x y}\right)\right)\right\} .
\end{aligned}
$$

This implies

$$
\begin{aligned}
\left|\frac{\partial}{\partial t} W_{t}^{\lambda}(x, y)\right| & \leq C_{\lambda} \frac{(x y)^{-\lambda}}{t^{1 / 2}} e^{-x^{2} / 16 t}\left(\frac{1}{t}+\frac{1}{x y}+\frac{x y}{t^{2}}+\frac{x^{2}+y^{2}}{t^{2}}+\frac{x^{2}+y^{2}}{x y t}\right) \\
& \leq C_{\lambda} \frac{(x y)^{-\lambda} x^{2}}{t^{5 / 2}} e^{-x^{2} / 16 t},
\end{aligned}
$$

since by the present assumptions on $x, y$ and $t$ we have

$$
\frac{1}{x y} \leq \frac{1}{t} \leq \frac{x^{2}+y^{2}}{t x y} \leq \frac{x^{2}+y^{2}}{t^{2}} \leq 2 \frac{x^{2}}{t^{2}} .
$$

Using again the assumptions $x y \geq t$ and $y<x / 2$ it follows that

$$
\begin{aligned}
\left(\int_{0}^{x y} t\left|\frac{\partial}{\partial t} W_{t}^{\lambda}(x, y)\right|^{2} d t\right)^{1 / 2} & \leq C_{\lambda}\left(\int_{0}^{x y} t\left|(x y)^{-\lambda} \frac{x^{2}}{t^{5 / 2}} e^{-x^{2} / 16 t}\right|^{2} d t\right)^{1 / 2} \\
& \leq C_{\lambda} \frac{1}{x^{2 \lambda+3}}\left(x y \int_{0}^{x y}\left(\frac{x^{2}}{t}\right)^{2 \lambda+5} e^{-x^{2} / 8 t} d t\right)^{1 / 2} \\
& \leq C_{\lambda} \frac{x y}{x^{2 \lambda+3}},
\end{aligned}
$$

and the last quantity can be easily estimated from above by $C_{\lambda} x^{-2 \lambda-1}$.

CASE 2: $x y<t$. Notice that by 5.2 we have

$$
\left(\int_{x y}^{\infty} t\left|\frac{\partial}{\partial t} W_{t}^{\lambda}(x, y)\right|^{2} d t\right)^{1 / 2} \leq \frac{C_{\lambda}}{\left(x^{2}+y^{2}\right)^{\lambda+1 / 2}} \leq C_{\lambda} x^{-2 \lambda-1} .
$$

Putting the two cases together produces

$$
\left(\int_{0}^{\infty} t\left|\frac{\partial}{\partial t} W_{t}^{\lambda}(x, y)\right|^{2} d t\right)^{1 / 2} \leq C_{\lambda} x^{-2 \lambda-1}, \quad 0<y<x / 2
$$

which is precisely what we needed.

The proof of Lemma 5.1 is finished. 
Lemma 5.2. Let $\lambda>-1 / 2$. There exist (positive) constants $a=a_{\lambda}$ and $c=c_{\lambda}$ such that

$$
\frac{\partial}{\partial t} W_{t}^{\lambda}(x, y) \leq-\frac{c}{t^{\lambda+3 / 2}}
$$

if either $0<x, y<a$ and $t \geq 1$, or $0<y<x$ and $x^{2} / t \leq a$.

Proof. The conclusion follows in a straightforward manner by combining (5.1) and 2.8.

Define the auxiliary square function

$$
\begin{aligned}
\mathfrak{g}_{\lambda, \operatorname{loc}}(f) & (x) \\
= & \left(\int_{0}^{\infty} t\left|\int_{x / 2}^{2 x}(x y)^{-\lambda} \frac{\partial}{\partial t} \mathcal{W}_{t}(x, y) f(y) d \mu_{\lambda}(y)\right|^{2} d t\right)^{1 / 2}, \quad x>0,
\end{aligned}
$$

which is the local part of a modification of the classical vertical square function.

Lemma 5.3. Let $\lambda \in \mathbb{R}$. Then, for each $\delta \in \mathbb{R}$, $\mathfrak{g}_{\lambda, \text { loc }}$ is bounded on $L^{p}\left(\mathbb{R}_{+}, x^{\delta} d x\right), 1<p<\infty$, and from $L^{1}\left(\mathbb{R}_{+}, x^{\delta} d x\right)$ into $L^{1, \infty}\left(\mathbb{R}_{+}, x^{\delta} d x\right)$.

Proof. Observe that the mapping $\mathfrak{g}_{0 \text {,loc }}$ is bounded on $L^{2}\left(\mathbb{R}_{+}, d x\right)$. This can be easily verified by invoking the well-known fact that the classical vertical $g$-function $\mathfrak{g}$ (restricted to $\mathbb{R}_{+}$and given by a formula analogous to that defining $\mathfrak{g}_{0, \text { loc }}$, but with the integration in $y$ from 0 to $\infty$ ) is bounded on $L^{2}\left(\mathbb{R}_{+}, d x\right)$, and then using classical Hardy inequalities.

From the $L^{2}$-boundedness of $\mathfrak{g}_{0, \text { loc }}$ we infer that the operator $\mathcal{G}$ assigning to an $f \in L^{2}\left(\mathbb{R}_{+}, d x\right)$ the function

$$
\mathbb{R}_{+} \ni x \mapsto \mathcal{G} f(x)=\left\{\int_{x / 2}^{2 x} \frac{\partial}{\partial t} \mathcal{W}_{t}(x, y) f(y) d y\right\}_{t>0}
$$

is bounded from $L^{2}\left(\mathbb{R}_{+}, d x\right)$ to the Bochner-Lebesgue space $L_{L^{2}(t d t)}^{2}\left(\mathbb{R}_{+}, d x\right)$. Moreover, $\mathcal{G}$ is associated, in the sense of Lemma 2.8, with the vector-valued kernel

$$
K(x, y)=\left\{\frac{\partial}{\partial t} \mathcal{W}_{t}(x, y)\right\}_{t>0} .
$$

This follows essentially by the known fact that $\mathfrak{g}$, viewed as a vector-valued Calderón-Zygmund operator, is associated with the same kernel. In addition, $K$ satisfies the standard estimates from Lemma 2.8 (even for all $x, y \in \mathbb{R}$ ), as is known and not hard to check.

Taking into account the above facts and applying Lemma 2.8 with $T=\mathcal{G}$ we see that the mapping $f(x) \mapsto x^{-\lambda} \mathcal{G}\left(y^{\lambda} f\right)(x)$ is a local vector-valued Calderón-Zygmund operator, hence, for every $\delta \in \mathbb{R}$, it extends to a bounded 
operator from $L^{p}\left(\mathbb{R}_{+}, x^{\delta} d x\right)$ to $L_{L^{2}(t d t)}^{p}\left(\mathbb{R}_{+}, x^{\delta} d x\right)$ for any $1<p<\infty$, and to a bounded operator from $L^{1}\left(\mathbb{R}_{+}, x^{\delta} d x\right)$ to $L_{L^{2}(t d t)}^{1, \infty}\left(\mathbb{R}_{+}, x^{\delta} d x\right)$.

Finally, notice that these boundedness results imply precisely the desired mapping properties of $\mathfrak{g}_{\lambda \text {,loc }}$ (the fact that the vector-valued bounded extensions correspond to $\mathfrak{g}_{\lambda, \text { loc }}$ given by $(5.3)$ follows by a standard density argument).

Having the above results we are ready to prove Theorem 2.5 .

Proof of Theorem 2.5. Making use of the triangle inequality for the norm $\|\cdot\|_{L^{2}(t d t)}$ and then applying Minkowski's integral inequality we get $g_{\lambda}(f)(x)$

$$
\begin{aligned}
\leq & \left\{\int_{0}^{x / 2}+\int_{2 x}^{\infty}\right\}\left(\int_{0}^{\infty} t\left|\frac{\partial}{\partial t} W_{t}^{\lambda}(x, y)\right|^{2} d t\right)^{1 / 2}|f(y)| d \mu_{\lambda}(y) \\
& +\int_{x / 2}^{2 x}\left(\int_{0}^{\infty} t\left|\frac{\partial}{\partial t} W_{t}^{\lambda}(x, y)-(x y)^{-\lambda} \frac{\partial}{\partial t} \mathcal{W}_{t}(x, y)\right|^{2} d t\right)^{1 / 2}|f(y)| d \mu_{\lambda}(y) \\
& +\left(\int_{0}^{\infty} t\left|\int_{x / 2}^{2 x}(x y)^{-\lambda} \frac{\partial}{\partial t} \mathcal{W}_{t}(x, y) f(y) d \mu_{\lambda}(y)\right|^{2} d t\right)^{1 / 2} .
\end{aligned}
$$

Then by Lemma 5.1 it follows that, for $x>0$,

$$
g_{\lambda}(f)(x) \leq C_{\lambda}\left(H_{0}^{2 \lambda}|f|(x)+H_{\infty}^{0}|f|(x)+\mathcal{N}|f|(x)+\mathfrak{g}_{\lambda, \text { loc }}(f)(x)\right),
$$

where $\mathcal{N}$ denotes the operator

$$
\mathcal{N} f(x)=\int_{x / 2}^{2 x} \frac{f(y)}{y} d y, \quad x>0 .
$$

Note that $\mathcal{N}$ is bounded on $L^{p}\left(\mathbb{R}_{+}, x^{\delta} d x\right)$ for each $1 \leq p \leq \infty$ and each $\delta \in \mathbb{R}$ (this can be easily verified by means of Jensen's inequality and Fubini's theorem, as in the case of the operator $N$ emerging in the proof of Theorem 2.3). Furthermore, by Lemma $5.3, \mathfrak{g}_{\lambda, \text { loc }}$ is bounded on $L^{p}\left(\mathbb{R}_{+}, x^{\delta} d x\right), 1<p<\infty$, and from $L^{1}\left(\mathbb{R}_{+}, x^{\delta} d x\right)$ into $L^{1, \infty}\left(\mathbb{R}_{+}, x^{\delta} d x\right)$, for each $\delta \in \mathbb{R}$.

Then, as in the proof of Theorem 2.3, by means of Lemmas 2.6 and 2.7 we conclude that $g_{\lambda}$ is bounded on $L^{p}\left(\mathbb{R}_{+}, x^{\delta} d x\right)$ when $1<p<\infty$ and $-1<\delta<(2 \lambda+1) p-1$, it is bounded from $L^{1}\left(\mathbb{R}_{+}, x^{\delta} d x\right)$ into $L^{1, \infty}\left(\mathbb{R}_{+}, x^{\delta} d x\right)$ when $-1<\delta \leq 2 \lambda$, and finally it is of restricted weak type $(p, p)$ with respect to $\left(\mathbb{R}_{+}, x^{\delta} d x\right)$ when $1 \leq p<\infty$ and $-1<\delta \leq(2 \lambda+1) p-1$. These facts justify the sufficiency parts in Theorem 2.5 .

We now prove the necessity parts. It suffices to show the following statements (we assume that $\lambda>-1 / 2,1 \leq p<\infty$ and the underlying space is $\left.\left(\mathbb{R}_{+}, x^{\delta} d x\right)\right)$. 
(A) If $g_{\lambda}$ is of restricted weak type $(p, p)$ then $-1<\delta \leq(2 \lambda+1) p-1$.

(B) $g_{\lambda}$ is not of weak type $(p, p)$ when $p>1$ and $\delta=(2 \lambda+1) p-1$.

(C) $g_{\lambda}$ is not of strong type $(1,1)$ if $-1<\delta \leq 2 \lambda$.

Let $f=\chi_{(a / 2, a)}$, where $a$ is the constant from Lemma 5.2. Then, according to that lemma,

$$
\begin{aligned}
g_{\lambda}(f)(x) & \geq\left(\int_{1}^{\infty} t\left|\int_{a / 2}^{a} \frac{\partial}{\partial t} W_{t}^{\lambda}(x, y) d \mu_{\lambda}(y)\right|^{2} d t\right)^{1 / 2} \\
& \geq c_{\lambda}\left(\int_{1}^{\infty} \frac{1}{t^{2 \lambda+2}}\left|\int_{a / 2}^{a} y^{2 \lambda} d y\right|^{2} d t\right)^{1 / 2} \\
& =c_{\lambda}, \quad 0<x<a .
\end{aligned}
$$

Suppose that $g_{\lambda}$ can be extended from $L^{2}\left(\mathbb{R}_{+}, d \mu_{\lambda}\right)$ to a restricted weak type $(p, p)$ operator on $\left(\mathbb{R}_{+}, x^{\delta} d x\right)$. For sufficiently small $\gamma>0$ we then have

$$
\int_{0}^{a} x^{\delta} d x \leq C_{p, \lambda} \gamma^{-p}\|f\|_{L^{p}\left(\mathbb{R}_{+}, x^{\delta} d x\right)}^{p} \leq C_{p, \lambda} \gamma^{-p},
$$

which implies $\delta>-1$.

On the other hand, using again Lemma 5.2, we get

$$
\begin{aligned}
g_{\lambda}(f)(x) & \geq c_{\lambda}\left(\int_{x^{2} / a}^{\infty} \frac{1}{t^{2 \lambda+2}}\left|\int_{0}^{1} f(y) d \mu_{\lambda}(y)\right|^{2} d t\right)^{1 / 2} \\
& \geq c_{\lambda} x^{-2 \lambda-1} \int_{0}^{1} f(y) d \mu_{\lambda}(y), \quad x>1,
\end{aligned}
$$

for any nonnegative measurable function $f$ on $\mathbb{R}_{+}$. Thus taking $f=\chi_{(1 / 2,1)}$ we have $g_{\lambda}(f)(x) \geq c_{\lambda} x^{-2 \lambda-1}$, provided that $x>1$. Consequently, if $g_{\lambda}$ can be extended from $L^{2}\left(\mathbb{R}_{+}, d \mu_{\lambda}\right)$ to a restricted weak type $(p, p)$ operator on $\left(\mathbb{R}_{+}, x^{\delta} d x\right)$, then

$$
\int_{1}^{\gamma^{-1 /(2 \lambda+1)}} x^{\delta} d x \leq C_{p, \lambda} \gamma^{-p}\|f\|_{L^{p}\left(\mathbb{R}_{+}, x^{\delta} d x\right)}^{p} \leq C_{p, \lambda} \gamma^{-p}
$$

for $\gamma>0$ small enough. This implies $\delta \leq(2 \lambda+1) p-1$ and (A) is justified.

In order to show (B) we use the estimate (5.4) and proceed as in the proof of the corresponding result in Theorem 2.1.

Considering (C), we argue as in the proofs of the parallel properties in Theorems 2.1 and 2.3 . We shall first see that $\mathfrak{g}_{\lambda \text {,loc }}$ is not bounded on $L^{1}\left(\mathbb{R}_{+}, x^{\delta} d x\right)$. Let $0<\varepsilon<1 / 2$ and assume that $1<y<1+\varepsilon, 1+2 \varepsilon<x<2$ 
and $t \geq 2(x-1)^{2}$. Then we have

$$
\frac{\partial}{\partial t} \mathcal{W}_{t}(x, y)=\frac{1}{2 \sqrt{\pi} t^{3 / 2}}\left(-1+\frac{(x-y)^{2}}{2 t}\right) e^{-(x-y)^{2} / 4 t} \leq-c t^{-3 / 2}
$$

for some $c>0$, because $(x-y)^{2} / 2 t \leq 9 / 16$ (see (3.6)). Hence, letting $f_{\varepsilon}=\chi_{(1,1+\varepsilon)}$, it follows that

$$
\begin{aligned}
\mathfrak{g}_{\lambda, \mathrm{loc}}\left(f_{\varepsilon}\right)(x) & =\left(\int_{0}^{\infty} t\left|\int_{x / 2}^{2 x} \frac{\partial}{\partial t} \mathcal{W}_{t}(x, y)(x y)^{-\lambda} f_{\varepsilon}(y) d \mu_{\lambda}(y)\right|^{2} d t\right)^{1 / 2} \\
& \geq c_{\lambda}\left(\int_{2(x-1)^{2}}^{\infty} \frac{d t}{t^{2}}\right)^{1 / 2}\left(\int_{1}^{1+\varepsilon} d y\right) \\
& \geq c_{\lambda} \frac{\varepsilon}{x-1}, \quad x \in(1+2 \varepsilon, 2) .
\end{aligned}
$$

Consequently,

$$
\begin{aligned}
\int_{0}^{\infty} \mathfrak{g}_{\lambda, \operatorname{loc}}\left(f_{\varepsilon}\right)(x) x^{\delta} d x & \geq \int_{1+2 \varepsilon}^{2} \mathfrak{g}_{\lambda, \operatorname{loc}}\left(f_{\varepsilon}\right)(x) x^{\delta} d x \geq c_{\lambda} \varepsilon \int_{1+2 \varepsilon}^{2} \frac{x^{\delta} d x}{x-1} \\
& \geq c_{\lambda, \delta} \varepsilon \log \frac{1}{2 \varepsilon} .
\end{aligned}
$$

Now, if $\mathfrak{g}_{\lambda, \text { loc }}$ were bounded on $L^{1}\left(\mathbb{R}_{+}, x^{\delta} d x\right)$ we would have

$$
c_{\lambda, \delta} \varepsilon \log \frac{1}{2 \varepsilon} \leq \int_{0}^{\infty} \mathfrak{g}_{\lambda, \operatorname{loc}}\left(f_{\varepsilon}\right)(x) x^{\delta} d x \leq C \int_{0}^{\infty} f_{\varepsilon}(x) x^{\delta} d x \leq C_{\delta} \varepsilon,
$$

which obviously cannot hold as $\varepsilon \rightarrow 0^{+}$. Thus $\mathfrak{g}_{\lambda, \text { loc }}$ is not bounded on $L^{1}\left(\mathbb{R}_{+}, x^{\delta} d x\right)$. On the other hand, by Lemma 5.1 and Minkowski's integral inequality we see that, for $x>0$,

$$
\begin{aligned}
\mathfrak{g}_{\lambda, \mathrm{loc}}\left(f_{\varepsilon}\right)(x) & \left(\int_{0}^{\infty} t\left|\int_{x / 2}^{2 x}\left(\frac{\partial}{\partial t}\left((x y)^{-\lambda} \mathcal{W}_{t}(x, y)\right)-\frac{\partial}{\partial t} W_{t}^{\lambda}(x, y)\right) f_{\varepsilon}(y) d \mu_{\lambda}(y)\right|^{2} d t\right)^{1 / 2} \\
& +\left(\int_{0}^{\infty} t\left|\int_{x / 2}^{2 x} \frac{\partial}{\partial t} W_{t}^{\lambda}(x, y) f_{\varepsilon}(y) d \mu_{\lambda}(y)\right|^{2} d t\right)^{1 / 2} \\
\leq & C_{\lambda}\left\{\int_{x / 2}^{2 x} \frac{\left|f_{\varepsilon}(y)\right|}{y} d y+\left(\int_{0}^{\infty} t\left|\int_{x / 2}^{2 x} \frac{\partial}{\partial t} W_{t}^{\lambda}(x, y) f_{\varepsilon}(y) d \mu_{\lambda}(y)\right|^{2} d t\right)^{1 / 2}\right\}
\end{aligned}
$$

This gives

$$
\mathfrak{g}_{\lambda, \mathrm{loc}}\left(f_{\varepsilon}\right)(x) \leq C_{\lambda}\left(\mathcal{N}\left|f_{\varepsilon}\right|(x)+g_{\lambda}\left(f_{\varepsilon}\right)(x)\right), \quad x \in(1+2 \varepsilon, 2) .
$$


Hence, taking into account weighted $L^{1}$-boundedness of the operator $\mathcal{N}$, if $g_{\lambda}$ were bounded on $L^{1}\left(\mathbb{R}_{+}, x^{\delta} d x\right)$ then (5.5) would hold, for all $0<\varepsilon<1 / 2$, a contradiction. Thus $g_{\lambda}$ is not bounded on $L^{1}\left(\mathbb{R}_{+}, x^{\delta} d x\right)$ and statement $(\mathrm{C})$ follows.

The proof of Theorem 2.5 is complete.

REMARK 5.4. Similar facts to those from Remark 3.2 are true for the corresponding $g$-function in the $\widetilde{\Delta}_{\lambda}$-setting. In particular, the relevant interval for $\widetilde{g}_{\lambda}$ is $(-\lambda p-1,(\lambda+1) p-1)$.

6. Operators related to the Poisson integral. Recall that the operators analyzed in the previous sections were defined by means of the heat kernel $W_{t}^{\lambda}(x, y)$. In this section we consider the maximal operator and a square function related to the Poisson kernel $P_{t}^{\lambda}(x, y)$ associated with $\Delta_{\lambda}$, $\lambda>-1 / 2$.

First of all, we shall compute $P_{t}^{\lambda}(x, y)$. By the principle of subordination,

$$
P_{t}^{\lambda}(x, y)=\int_{0}^{\infty} W_{t^{2} / 4 u}^{\lambda}(x, y) \frac{e^{-u} d u}{\sqrt{\pi u}} .
$$

Then an application of the integral formula 4.2 leads to

$$
\begin{aligned}
& P_{t}^{\lambda}(x, y) \\
& \quad=\mathcal{C}(\lambda) \frac{t}{\left(x^{2}+y^{2}+t^{2}\right)^{\lambda+1}}{ }_{2} F_{1}\left(\frac{\lambda+1}{2}, \frac{\lambda+2}{2} ; \frac{2 \lambda+1}{2} ;\left(\frac{2 x y}{x^{2}+y^{2}+t^{2}}\right)^{2}\right),
\end{aligned}
$$

with $\mathcal{C}(\lambda)=2 \pi^{-1 / 2} \Gamma(\lambda+1) / \Gamma(\lambda+1 / 2)$. We now transform the above expression in order to see the exact behavior of the Poisson kernel. Using the formula (cf. [12, (9.5.3)])

$$
{ }_{2} F_{1}(\alpha, \beta ; \gamma ; z)=(1-z)^{\gamma-\alpha-\beta}{ }_{2} F_{1}(\gamma-\alpha, \gamma-\beta ; \gamma ; z),
$$

valid for $z<1$, one gets

$$
\begin{aligned}
P_{t}^{\lambda}(x, y)= & \mathcal{C}(\lambda) \frac{t\left(x^{2}+y^{2}+t^{2}\right)^{1-\lambda}}{\left[(x+y)^{2}+t^{2}\right]\left[(x-y)^{2}+t^{2}\right]} \\
& \times{ }_{2} F_{1}\left(\frac{\lambda}{2}, \frac{\lambda-1}{2} ; \frac{2 \lambda+1}{2} ;\left(\frac{2 x y}{x^{2}+y^{2}+t^{2}}\right)^{2}\right) .
\end{aligned}
$$

We observe that the values of the last ${ }_{2} F_{1}$ function are separated from 0 and $\infty$; this is because the function $z \mapsto{ }_{2} F_{1}(\lambda / 2,(\lambda-1) / 2 ; \lambda+1 / 2 ; z)$ is continuous on $[0,1)$, has value 1 at $z=0$ (see [12, (9.1.1)]), its limit as $z \rightarrow 1^{-}$exists and is positive (cf. [12, Section 9.3]) and, finally, there are no zeroes in $(0,1)$ since the Poisson kernel is strictly positive (this is of course a consequence of the same property for the heat kernel). Thus we obtain the following. 
Proposition 6.1. Let $\lambda>-1 / 2$. There exists a constant $C_{\lambda}$ such that, for all $t, x, y>0$,

$$
\frac{C_{\lambda}^{-1} t}{\left(x^{2}+y^{2}+t^{2}\right)^{\lambda}\left[(x-y)^{2}+t^{2}\right]} \leq P_{t}^{\lambda}(x, y) \leq \frac{C_{\lambda} t}{\left(x^{2}+y^{2}+t^{2}\right)^{\lambda}\left[(x-y)^{2}+t^{2}\right]} .
$$

It should not be surprising that in the case $\lambda=0$ the Poisson kernel has a particularly simple form. Notice that the first parameter in the ${ }_{2} F_{1}$ expressing $P_{t}^{0}(x, y)$ is zero, consequently the hypergeometric function is identically equal to 1 and we get

$$
P_{t}^{0}(x, y)=\frac{1}{\pi}\left(\frac{t}{(x-y)^{2}+t^{2}}+\frac{t}{(x+y)^{2}+t^{2}}\right) .
$$

This shows that $P_{t}^{0}(x, y)=\mathcal{P}_{t}(x, y)+\mathcal{P}_{t}(x,-y)$, with $\mathcal{P}_{t}$ being the classical Poisson kernel. Clearly, the same identity could be deduced immediately from the analogous, already discussed in Section 4, connection between $W_{t}^{0}$ and $\mathcal{W}_{t}$.

Apart from the maximal operator $P_{*}^{\lambda}$ we consider the Littlewood-Paley type square function

$$
\partial_{\lambda}(f)(x)=\left(\int_{0}^{\infty} t\left|\frac{\partial}{\partial t} P_{t}^{\lambda} f(x)\right|^{2} d t\right)^{1 / 2} .
$$

It turns out that various boundedness results for the two operators can be deduced, in a straightforward manner, by means of Theorems 2.1 and 2.5 . A key fact here is that $P_{*}^{\lambda} f$ and $\partial_{\lambda}(f)$ can be controlled pointwise by $W_{*}^{\lambda} f$ and $g_{\lambda}(f)$, respectively.

Proposition 6.2. Let $\lambda>-1 / 2$. Then, for sufficiently regular functions $f$,

$$
P_{*}^{\lambda} f(x) \leq W_{*}^{\lambda} f(x), \quad \partial_{\lambda}(f)(x) \leq \sqrt{2} g_{\lambda}(f)(x), \quad x>0 .
$$

Proof. By subordination and Fubini's theorem,

$$
\begin{aligned}
P_{*}^{\lambda} f(x) & =\sup _{t>0}\left|\int_{0}^{\infty} W_{t^{2} / 4 u}^{\lambda} f(x) \frac{e^{-u} d u}{\sqrt{\pi u}}\right| \\
& \leq \int_{0}^{\infty} \sup _{t>0}\left|W_{t^{2} / 4 u}^{\lambda} f(x)\right| \frac{e^{-u} d u}{\sqrt{\pi u}}=W_{*}^{\lambda} f(x) .
\end{aligned}
$$

Next, again by subordination,

$$
\begin{aligned}
\partial_{\lambda}(f)(x) & =\left(\int_{0}^{\infty} t\left|\frac{\partial}{\partial t} \int_{0}^{\infty} W_{t^{2} / 4 u}^{\lambda} f(x) \frac{e^{-u} d u}{\sqrt{\pi u}}\right|^{2} d t\right)^{1 / 2} \\
& =\left\|\int_{0}^{\infty}\left(\partial_{t} W^{\lambda}\right)_{t^{2} / 4 u} f(x) \frac{t}{2 u} \frac{e^{-u} d u}{\sqrt{\pi u}}\right\|_{L^{2}(t d t)},
\end{aligned}
$$


where $\left(\partial_{t} W^{\lambda}\right)_{t^{2} / 4 u} f(x)$ is the derivative in $s$ of $W_{s}^{\lambda} f(x)$, taken at the point $s=t^{2} / 4 u$. Then applying Minkowski's integral inequality and changing the variable we obtain

$$
\begin{aligned}
\partial_{\lambda}(f)(x) & \leq \int_{0}^{\infty}\left\|t\left(\partial_{t} W^{\lambda}\right)_{t^{2} / 4 u} f(x)\right\|_{L^{2}(t d t)} \frac{e^{-u} d u}{2 u \sqrt{\pi u}} \\
& =\sqrt{2} \int_{0}^{\infty} \frac{e^{-u} d u}{\sqrt{\pi u}}\left(\int_{0}^{\infty} s\left|\frac{\partial}{\partial s} W_{s}^{\lambda} f(x)\right|^{2} d s\right)^{1 / 2} \\
& =\sqrt{2} g_{\lambda}(f)(x) .
\end{aligned}
$$

The proof is complete.

Thus, by Proposition 6.2 and Theorems 2.1 and 2.5. we obtain strong type, weak type and restricted weak type mapping properties of the Poisson integral based operators. However, since the subordination principle means a kind of averaging, it is far from obvious whether these results are sharp in respect of admissible powers $\delta$. In the case of $P_{*}^{\lambda}$ the precise behavior of the corresponding kernel is known (Proposition 6.1) and is relatively simple. Hence we can easily obtain the following estimates, similar to those from Section 3 and now involving the Poisson kernel.

Proposition 6.3. Let $\lambda>-1 / 2$. There exists $c_{\lambda}>0$ such that

$$
P_{t}^{\lambda}(x, y) \geq c_{\lambda} \frac{t}{t^{2}+(x-y)^{2}}, \quad t \leq 1 / 2, x, y \in(1,2),
$$

and such that, for every nonnegative measurable function $f$ on $(0, \infty)$, we have

$$
P_{*}^{\lambda} f(x) \geq c_{\lambda} \int_{0}^{\infty} \frac{f(y)}{\left(1+y^{2}\right)^{\lambda+1}} d \mu_{\lambda}(y), \quad x \in(0,1),
$$

and

$$
P_{*}^{\lambda} f(x) \geq c_{\lambda} x^{-2 \lambda-1} \int_{0}^{x} f(y) d \mu_{\lambda}(y), \quad x \in(0, \infty) .
$$

Now, using the arguments from the proofs of Theorems 2.1 and 2.5 to justify necessity parts, we obtain sharp results for the Poisson integral maximal operator stated in Theorem 2.2 .

Proving similar results for $\widetilde{\partial}_{\lambda}$ requires a deeper and more subtle analysis, which is beyond the scope of this paper. We only mention that in order to obtain suitable kernel estimates one has to deal with terms involving hypergeometric functions with different parameters and examine essential cancellations occurring between those terms (for that purpose it seems to be more convenient to use, instead of ${ }_{2} F_{1}$, the integral representation (6.1) of $P_{t}^{\lambda}(x, y)$ in terms of $\left.W_{t}^{\lambda}(x, y)\right)$. 
Acknowledgments. The authors gratefully acknowledge the hospitality of Professor José Luis Torrea and the Department of Mathematics at Universidad Autónoma de Madrid. A part of the investigation of the first, second and fourth-named authors during the preparation of this paper was made while they were visiting Universidad Autónoma de Madrid. The thirdnamed author was partially working on this paper during a postdoctoral visiting position period at the Department of Mathematics of Universidad Autónoma de Madrid, October 2005-March 2006.

The first-named author was partially supported by MTM2007/65609. The second and the fourth-named authors were partially supported by grants from CONICET and ANPCyT of Argentina and from Universidad Nacional del Litoral. The third-named author was partially supported by MNiSW Grant N201 054 32/4285.

\section{References}

[1] K. F. Andersen and R. A. Kerman, Weighted norm inequalities for generalized Hankel conjugate transformations, Studia Math. 71 (1981), 15-26.

[2] K. F. Andersen and B. Muckenhoupt, Weighted weak type Hardy inequalities with applications to Hilbert transforms and maximal functions, ibid. 77 (1982), 9-26.

[3] J. J. Betancor, D. Buraczewski, J. C. Fariña, M. T. Martínez, and J. L. Torrea, Riesz transforms related to Bessel operators, Proc. Roy. Soc. Edinburgh Sect. A 137 (2007), 701-725.

[4] J. J. Betancor, J. C. Fariña, M. T. Martínez, and J. L. Torrea, Riesz transform and $g$-function associated with Bessel operators and their appropriate Banach spaces, Israel J. Math. 157 (2007), 259-282.

[5] J. J. Betancor and K. Stempak, Relating multipliers and transplantation for FourierBessel expansions and Hankel transform, Tohoku Math. J. 53 (2001), 109-129.

[6] - - -, On Hankel conjugate functions, Studia Sci. Math. Hungar. 41 (2004), 59-91.

[7] W. R. Bloom and Z. Xu, Maximal functions on Chébli-Trimèche hypergroups, Infin. Dimens. Anal. Quantum Probab. Relat. Top. 3 (2000), 403-434.

[8] A. Chicco Ruiz and E. Harboure, Weighted norm inequalities for heat-diffusion Laguerre's semigroups, Math. Z. 257 (2007), 329-354.

[9] E. Harboure, C. Segovia, J. L. Torrea, and B. Viviani, Power weighted $L^{p}$-inequalities for Laguerre-Riesz transforms, Ark. Mat. 46 (2008), 285-313.

[10] R. A. Kerman, Generalized Hankel conjugate transformations on rearrangement invariant spaces, Trans. Amer. Math. Soc. 232 (1977), 111-130.

[11] —, Boundedness criteria for generalized Hankel conjugate transformations, Canad. J. Math. 30 (1978), 147-153.

[12] N. N. Lebedev, Special Functions and Their Applications, Dover Publ., New York, 1972.

[13] B. Muckenhoupt and E. M. Stein, Classical expansions and their relation to conjugate harmonic functions, Trans. Amer. Math. Soc. 118 (1965), 17-92.

[14] A. Nowak and K. Stempak, Weighted norm inequalities for the Hankel transform transplantation operator, Tohoku Math. J. 58 (2006), 277-301. 
[15] A. P. Prudnikov, Yu. A. Brychkov, and O. I. Marichev, Integrals and Series, Volume 2: Special Functions, Gordon and Breach, 1986.

[16] E. M. Stein, Topics in Harmonic Analysis Related to the Littlewood-Paley Theory, Princeton Univ. Press, Princeton, 1970.

[17] K. Stempak, The Littlewood-Paley theory for the Fourier-Bessel transform, Preprint 45, Math. Inst., Univ. of Wrocław, 1985.

[18] G. N. Watson, A Treatise on the Theory of Bessel Functions, Cambridge Univ. Press, Cambridge, 1966.

Jorge J. Betancor

Departamento de Análisis Matemático

Universidad de la Laguna

Campus de Anchieta

Avda. Astrofísico Francisco Sánchez, s/n

38271 La Laguna (Sta. Cruz de Tenerife), Spain

E-mail: jbetanco@ull.es
Eleonor Harboure, Beatriz Viviani

IMAL-FIQ

CONICET-Universidad Nacional del Litoral

Guemes 3450

3000 Santa Fe, Argentina

E-mail: harbour@ceride.gov.ar viviani@ceride.gov.ar

Adam Nowak

Instytut Matematyki i Informatyki

Politechnika Wrocławska

Wybrzeże Wyspiańskiego 27

50-370 Wrocław, Poland

E-mail: Adam.Nowak@pwr.wroc.pl 